\title{
LERAY FUNCTOR AND COHOMOLOGICAL CONLEY INDEX FOR DISCRETE DYNAMICAL SYSTEMS
}

\author{
MARIAN MROZEK
}

\begin{abstract}
We introduce the Leray functor on the category of graded modules equipped with an endomorphism of degree zero and we use this functor to define the cohomological Conley index of an isolated invariant set of a homeomorphism on a locally compact metric space. We prove the homotopy and additivity properties for this index and compute the index in some examples. As one of applications we prove the existence of nonconstant, bounded solutions of the Euler approximation of a certain system of ordinary differential equations.
\end{abstract}

\section{INTRODUCTION}

The problem of the construction of the Conley index for the case of discrete time dynamical systems was posed already in Conley's book [Co]. When studying this problem in detail one can see that most steps of the continuous case construction (in particular the definition and existence theorem for index pairs) can be carried over without difficulties to the discrete case. The only serious obstacle is the lack of homotopy along trajectories of the flow. Because of this, the homotopy type of an index pair and its homology or cohomology are not invariants.

In a recent paper Robbin and Salamon [RS] chose the shape theory to overcome this difficulty (in smooth setting) and define the shape index as the shape of the one-point compactification of the unstable manifold of an isolated invariant set (with some special intrinsic topology). This construction is very interesting because it shows the close relation between the Conley index and the topology of the unstable manifold of an isolated invariant set. On the other hand, the shape index seems not to be a convenient tool in applications, because of the presence of inverse limits appearing in its construction.

In the present paper we propose a construction of the cohomological Conley index for discrete dynamical systems, which goes along Conley lines whenever it is possible, with certain modifications taken from [Mr2]. The problem of lack of the trajectory-defined homotopy is overcome by considering not only

Received by the editors April 22, 1988.

1980 Mathematics Subject Classification (1985 Revision). Primary 58F35; Secondary 34C35, $54 \mathrm{H} 20$.

Key words and phrases. Conley index, Leray endomorphism, isolated invariant set. 
index pairs but also index maps associated with every index pair (cf. [Mr1]) and by introducing a new functor (the Leray functor) from the category of graded modules equipped with an endomorphism of degree zero in its proper subcategory of graded modules equipped with an isomorphism. The definition of this functor is based on the notion of a generalized kernel used by Leray [Le] to define the generalized Lefschetz number as well as on the dual notion of the generalized image.

The index takes the form of a graded module with a distinguished isomorphism. The isomorphism has no counterpart in the case of the shape index. Therefore our index distinguishes between isolated invariant sets which cannot be differentiated by the index of Robbin and Salamon.

In this paper we consider the case of a discrete dynamical system given by a homeomorphism of a locally compact metric space. The generalization of the theory presented to the case of a continuous map (discrete semidynamical system) is straightforward. Removing the local compactness assumption in the spirit of Rybakowski [Ry] is also possible, through some different techniques are necessary (see $[M R]$ ).

We use the Alexander-Spanier cohomology only because of its nice excision properties. Other homology or cohomology theories can also be used, but then certain extra assumptions as in the case of regular index pairs (see [Mr1]) have to be added to the definition of the index pair.

The organization of the paper is as follows. $\S 1$ contains preliminaries. In $\S 2$ we present the main results of the paper. The indices of a hyperbolic fixed point and a hyperbolic periodic point are computed in $\S 3$. The Leray functor and its properties are studied in $\S 4$. Next we prove the existence of index pairs in the discrete case. In $\S 6$ the index is constructed. The main properties of the index are proved in $\S 7$. In $\S 8$ we present some examples.

\section{Preliminaries}

$\mathbf{R}, \mathbf{Z}, \mathbf{Z}^{+}, \mathbf{Z}^{-}$, and $\mathbf{N}$ will denote the sets of real, integer, nonnegative integer, nonpositive integer, and natural numbers, respectively. $\Xi$ will stand for a fixed ring with unity. For a topological space $X$ and a subset $A \subseteq X$ the notation $\operatorname{int}_{X} A, \mathrm{cl}_{X} A, \mathrm{bd}_{X} A$ will be used for the interior, the closure, and the boundary of $A$ in $X$, respectively. If this causes no misunderstanding, we shall drop the subscript $X$ in the above notation.

We will frequently consider pairs of topological spaces of the form $\left(X_{1}, X_{2}\right)$ without assuming that $X_{2} \subseteq X_{1}$. The special case $X_{2} \subseteq X_{1}$ will be referred to as a topological pair. In order to simplify the notation we will usually denote pairs of spaces by single capital letters. In such a situation the first or the second element of the pair will be denoted by adding to the letter the subscript 1 or 2 , respectively. Thus if $P$ is a pair of spaces then $P=\left(P_{1}, P_{2}\right)$, where $P_{1}, P_{2} \in$ Top. 
We make a general assumption that whenever a pair $P=\left(P_{1}, P_{2}\right)$ of subsets of a topological space $X$ appears in a notation making sense for topological pairs only, then $P$ should be considered as the pair $\left(P_{1}, P_{1} \cap P_{2}\right)$.

The notation used for spaces will be extended in a natural way to pairs of spaces. Thus if $P, Q$ are pairs of subspaces of a topological space $X$ and $f: X \rightarrow X$ is a mapping, then $f(P):=\left(f\left(P_{1}\right), f\left(P_{2}\right)\right)$. Similarly, $P \subseteq Q$ will mean $P_{i} \subseteq Q_{i}$ for $i=1,2$, int $P$ will denote the pair (int $P_{1}$, int $P_{2}$ ), etc. We shall identify a single space $X$ with the pair $(X, \varnothing)$.

By a map $f: P \rightarrow Q$ of pairs $P, Q$, we mean a continuous mapping $f: P_{1} \rightarrow Q_{1}$ such that $f\left(P_{1} \cap P_{2}\right) \subseteq Q_{1} \cap Q_{2}$, which is equivalent to $f\left(P_{1} \cap P_{2}\right) \subseteq$ $Q_{2}$.

Note that the identity map id: $P_{1} \ni x \rightarrow x \in P_{1}$ can be obviously considered as a map id: $P \rightarrow P$ of the pair $P=\left(P_{1}, P_{2}\right)$ into itself.

If $R, S$ are pairs such that $R \subseteq P, S \subseteq Q$, and $f: P_{1} \rightarrow Q_{1}$ is such that $f(R) \subseteq S$, then one can consider the map

$$
R_{1} \ni x \rightarrow f(x) \in S_{1},
$$

which maps $R_{1} \cap R_{2}$ into $S_{1} \cap S_{2}$. This map will be called the contraction of $f$ to the pair of pairs $(R, S)$ and denoted by $f_{R, S}$.

If $R, S$ are pairs such that $R \subseteq S$ then $i_{R, S}$ will stand for the contraction of the identity id: $S \rightarrow S$ to the pair of pairs $(R, S)$. It will be called the inclusion of pair $R$ into pair $S$.

In part of the paper the language of multivalued maps will be used. Recall that a multivalued (m.v.) map $\varphi: X \rightarrow Y$ is a mapping which assigns to every $x \in X$ some nonempty, compact subset $\varphi(x)$ of $Y$. The image of a subset $A \subseteq X$ under $\varphi$ is $\varphi(A):=\bigcup\{\varphi(x) \mid x \in A\}$. The m.v. map $\varphi$ is called uppersemicontinuous (u.s.c.) iff for every $x \in X$ and for every neighborhood $U$ of $\varphi(x)$ there exists a neighborhood $V$ of $x$ such that $\varphi(y) \subseteq U$ for all $y \in V$.

The following well-known property of m.v. maps will be needed.

Proposition 1.1. If $\varphi: X \rightarrow Y$ is u.s.c. and $K \subseteq X$ is compact then $\varphi(K)$ is compact.

$\mathscr{E}$ will denote the category of graded modules over the ring $\Xi$ and homomorphisms of degree zero. If $E, F \in \mathscr{E}$ then $\mathscr{E}(E, F)$ will stand for the set of all morphisms from $E$ to $F$ in $\mathscr{E}$. Thus, if $E, F \in \mathscr{E}, \varphi \in \mathscr{E}(E, F)$, then $E=\left\{E_{n}\right\}, F=\left\{F_{n}\right\}, \varphi=\left\{\varphi_{n}\right\}$, where $E_{n}, F_{n}$ are modules over $\Xi$ and $\varphi_{n}: E_{n} \rightarrow F_{n}$ are homomorphisms.

For $E_{1}, E_{2} \in \mathscr{E}$, the product of $E_{1}, E_{2}$ will be denoted by $E_{1} \times E_{2}$. If $E_{i}, F_{i} \in \mathscr{E}$ for $i=1,2$ and $\varphi_{i} \in \mathscr{E}\left(E_{i}, F_{i}\right)$ then $\varphi_{1} \times \varphi_{2}: E_{1} \times E_{2} \rightarrow F_{1} \times F_{2}$ will stand for the product of the maps $\varphi_{1}, \varphi_{2}$.

The functor of the Alexander-Spanier cohomology with coefficients in $\Xi$ will be considered as a functor $H^{*}:$ Top $_{2} \rightarrow \mathscr{E}$, where Top 2 stands for the category of topological pairs. Since the coefficient ring $\Xi$ is fixed, we shall omit it in the notation of the cohomology module. If $P=\left(P_{1}, P_{2}\right)$ is an arbitrary pair of 
subspaces of a topological space $X$, then, according to our general assumption, we put $H^{*}(P):=H^{*}\left(P_{1}, P_{1} \cap P_{2}\right)$.

The category $\mathscr{E} E$ of graded modules equipped with an endomorphism is defined as follows. The objects of the category $\mathscr{E} E$ are all pairs $(E, e)$, where $E \in \mathscr{E}$ and $e \in \mathscr{E}(E, E)$ is an endomorphism. The morphisms from $(E, e) \in$ $\mathscr{E} E$ to $(F, f) \in \mathscr{E} E$ are all maps $\varphi \in \mathscr{E}(E, F)$ such that the diagram

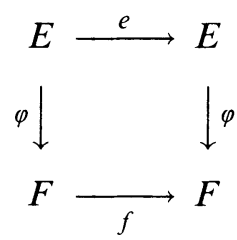

commutes. We will briefly write $\varphi:(E, e) \rightarrow(F, f)$ to denote that $\varphi$ is a morphism from $(E, e)$ to $(F, f)$.

In the sequel we will identify the isomorphic objects of $\mathscr{E} E$. Note that if $\varphi$ in (1.1) is an isomorphism, then $e, f$ are known as equivalent (similar) endomorphisms. In some cases a classification of equivalent endomorphisms can be given by means of the Jordan form of their matrices.

We define the categories $\mathscr{E} M$ and $\mathscr{E} I$ as the full subcategories of $\mathscr{E} E$ consisting of graded modules equipped with a mono- and isomorphism, respectively.

The object $(E, e) \in \mathscr{E} E$ such that $E_{n}=0$ and $e_{n}=0$ for all $n$ will be called the zero object of $\mathscr{E} E$ (or briefly zero) and it will be denoted by 0 .

We will consider the category $\mathscr{E}$ as a subcategory of $\mathscr{E} I$ using the natural functorial embedding

$$
\mathscr{E} \ni E \rightarrow(E, \mathrm{id}) \in \mathscr{E} I
$$

Thus we have

$$
\mathscr{E} \subseteq \mathscr{E} I \subseteq \mathscr{E} M \subseteq \mathscr{E} E
$$

\section{Main Results}

Let $(X, e)$ denote a fixed, locally compact metric space. By a discrete dynamical system on $X$ we mean a fixed homeomorphism $f: X \rightarrow X$. We will study isolated invariant sets of $f$. The set $K$ is invariant if for each $x \in K$ the trajectory of $x$, i.e. $\left\{f^{n}(x) \mid n \in \mathbf{Z}\right\}$, is contained in $K$. In other words, $K$ is invariant iff $f(K)=K . K$ is said to be an isolated invariant set if it is a compact invariant set which is the largest invariant set in its neighborhood $N$. In such case $N$ is said to isolate $K$. If $N$ is additionally compact, it is called an isolating neighborhood for $K$.

Note that the empty set is a trivial example of an isolated invariant set, which is also an isolating neighborhood of itself.

We assume an isolated invariant set $K$ and its fixed isolating neighborhood $N$ are given. 
$A \subseteq N$ is called positively invariant with respect to $N$ iff $A \cap f^{-1}(N) \subseteq$ $f^{-1}(A)$.

The main tool in constructing the Conley index for flows is the index pair. To define its discrete counterpart, we introduce the sets

$$
\begin{aligned}
\operatorname{Inv}^{+} N & :=\left\{x \in X \mid \forall i \in \mathbf{Z}^{+} f^{i}(x) \in N\right\}, \\
\operatorname{Inv}^{-} N & :=\left\{x \in X \mid \forall i \in \mathbf{Z}^{-} f^{i}(x) \in N\right\}, \\
\operatorname{Inv} N & :=\operatorname{Inv}^{-} N \cap \operatorname{Inv}^{+} N,
\end{aligned}
$$

which will be called the positively invariant, the negatively invariant, and the invariant part of $N$ (relative to $f$ ), respectively. Hence the set $A$ is invariant iff Inv $A=A$. Similarly, we say that $A$ is positively invariant (negatively invariant $)$ iff $\mathrm{Inv}^{+} A=A\left(\mathrm{Inv}^{-} A=A\right)$.

Definition 2.1. The pair $P=\left(P_{1}, P_{2}\right)$ of compact subsets of $N$ will be called an index pair of $K$ in $N$ (with respect to $f$ ) iff the following three conditions are satisfied.

(2.1) $P_{1}, P_{2}$ are positively invariant with respect to $N$;

(2.2) $\operatorname{Inv}^{-} N \subseteq \operatorname{int}_{N} P_{1}, \operatorname{Inv}^{+} N \subseteq N \backslash P_{2}$;

(2.3) $P_{1} \backslash P_{2} \subseteq \operatorname{int} N \cap f^{-1}($ int $N)$.

The family of all index pairs in $N$ will be denoted by $\operatorname{IP}(N, f)$ or simply by $\operatorname{IP}(N)$.

Three simple properties of index pairs are summarized in the following.

\section{Proposition 2.2.}

(2.4) $P \in \operatorname{IP}(N) \Rightarrow \operatorname{Inv} N \subseteq \operatorname{int}\left(P_{1} \backslash P_{2}\right)$.

(2.5) $P, Q \in \operatorname{IP}(N) \Rightarrow P \cap Q \in \operatorname{IP}(N)$,

(2.6) $x \in P_{1}, f(x) \notin N \Rightarrow x \in P_{2}$.

One can put forward other, slightly less restrictive definitions of the index pair. The above definition is convenient for us, because it is handy in proofs and not overrestrictive, as the following shows.

Theorem 2.3 (see also [RS and Mr1]). For every neighborhood $W$ of $K$ there exists an index pair $P$ in $N$ such that $P_{1} \backslash P_{2} \subseteq W$. (For proof see $\S 5$.)

As in the continuous case, we shall use index pairs to construct the cohomological Conley index in our case. Unfortunately, we cannot take $H^{*}\left(P_{1}, P_{2}\right)$ as the index, since, unlike the case of the flow, this cohomology does depend on the particular index pair (cf. [Mr1]). To overcome this difficulty we need two more notions: the index map and the Leray functor.

For $P \in \operatorname{IP}(N)$ we define

$$
\begin{aligned}
& S(P):=\left(P_{1} \cup f\left(P_{2}\right), P_{2} \cup f\left(P_{2}\right)\right), \\
& T(P):=T_{N}(P):=\left(P_{1} \cup(X \backslash \text { int } N), P_{2} \cup(X \backslash \text { int } N)\right) .
\end{aligned}
$$


Proposition 2.4. Assume $P \in \operatorname{IP}(N)$. Then

(2.7) $f(P) \subseteq S(P) \subseteq T(P)$,

(2.8) inclusions $i_{P, S(P)}, i_{S(P), T(P)}, i_{P, T(P)}$ induce isomorphisms in the Alexander-Spanier cohomology.

Proof. Assume $x \in P_{i}, f(x) \notin P_{i}$. Then, by (2.1), $f(x) \notin N$ and by (2.6), $x \in P_{2}$, i.e., $f(x) \in f\left(P_{2}\right) \subseteq P_{i} \cup f\left(P_{2}\right)$. This proves the first inclusion in (2.7). The second inclusion follows from (2.1).

We have

$$
\begin{gathered}
\left(P_{1} \cup f\left(P_{2}\right)\right) \backslash\left(P_{2} \cup f\left(P_{2}\right)\right)=P_{1} \backslash P_{2} \backslash f\left(P_{2}\right)=P_{1} \backslash P_{2}, \\
T\left(P_{1}\right) \backslash T\left(P_{2}\right)=\left(P_{1} \backslash P_{2}\right) \cap \operatorname{int} N=P_{1} \backslash P_{2},
\end{gathered}
$$

and (2.8) follows from the strong excision property of the Alexander-Spanier cohomology (see [Sp, Chapter 6.5, Theorem 5]).

Formula (2.7) shows that the contraction of $f$ to the pair of pairs $(P, T(P))$ is well defined. Introduce the notation

$$
f_{P}:=f_{P, T(P)}, \quad i_{P}:=i_{P, T(P)} .
$$

Since by $(2.8), H^{*}\left(i_{P}\right)$ is an isomorphism, we can pose the following:

Definition 2.5. The endomorphism $H^{*}\left(f_{P}\right) \circ H^{*}\left(i_{P}\right)^{-1}$ of $H^{*}(P)$ will be called the index map associated with the index pair $P$ and denoted by $I_{P}$.

The index map was first introduced in [Mr1].

In $\S 4$ we shall construct a functor $L: \mathscr{E} E \rightarrow \mathscr{E} I$, which we call the Leray functor, which restricted to $\mathscr{E} I$ is an identity (see Proposition 4.1).

Applying the Leray functor to $\left(H^{*}(P), I_{P}\right)$ we obtain an object of $\mathscr{E} E$, which will be called the Leray reduction of the Alexander-Spanier cohomology of the index pair $P$.

Now we are able to formulate the main results of the paper.

Theorem 2.6. Assume $f: X \rightarrow X$ is a homeomorphism and $K$ is an isolated invariant set with respect to $f$. Then for all $N, M$ isolating neighborhoods of $K$ and $P \in \operatorname{IP}(N), Q \in \operatorname{IP}(M)$ the Leray reductions of Alexander-Spanier cohomologies $L\left(H^{*}(P), I_{P}\right)$ and $L\left(H^{*}(Q), I_{Q}\right)$ are isomorphic (the proof will be given in $\S 6$ ).

Corollary 2.7. The Leray reduction of the Alexander-Spanier cohomology of an index pair of an isolated invariant set $K$ depends only on $K$.

Definition 2.8. The common value $L\left(H^{*}(P), I_{P}\right)$ for all index pairs $P$ of $K$ will be called the cohomological Conley index of $K$ (or briefly the index of $K$ ) and denoted by $C(K, f)$, or simply by $C(K)$ when $f$ is clear from context.

Obviously, $C(K)$ is a graded module over the ring $\Xi$ equipped with an endomorphism of degree zero. The $n$th component of $C(K)$ will be denoted 
by $C_{n}(K)$. Thus $C(K)=\left\{C_{n}(K)\right\}$, where $C_{n}(K)$ is a module over $\Xi$ with an endomorhpism.

The reader familiar with the Conley index for flows should note that if $f$ is the time-one map of a flow and $K$ is an isolated invariant set, then there exists an index pair $P$ both in the flow and homeomorphism sense. Moreover, $f_{P}$ is homotopic to $i_{P}$, thus $I_{p}$ is an identity. Consequently

$$
C(K)=L\left(H^{*}(P), \mathrm{id}\right)=\left(H^{*}(P), \mathrm{id}\right)=H^{*}(P) .
$$

Hence we have the following.

Theorem 2.9. The cohomological Conley index of an isolated invariant set of a flow coincides with the corresponding cohomological Conley index of the time-one map of this flow.

Hence our index can be considered as a generalization of the cohomological Conley index for flows.

The empty pair $(\varnothing, \varnothing)$ is obviously an index pair of the empty set. Since $H^{*}(\varnothing, \varnothing)=0$ and $L(0)=0$ (see Proposition 4.6) we get, as in the continuous case, the following important property of the Conley index.

Proposition 2.10. The Conley index of the empty set is zero. In other words, a nonzero Conley index implies a nonempty isolated invariant set.

This means that our index can be used to establish the existence of nonempty isolated invariant sets. We shall need, however, some tools for computing the index. As in the case of a flow, we have the continuation and additivity properties.

Theorem 2.11 (homotopy (continuation) property). Assume $\Lambda \subseteq \mathbf{R}$ is a compact interval and $f: \Lambda \times X \rightarrow X$ is a continuous map such that for each $\lambda \in \Lambda$

$$
f_{\lambda}: X \ni x \rightarrow f(\lambda, x) \in X
$$

is a homeomorphism and $N$ is an isolating neighborhood with respect to $f_{\lambda}$. Then $C\left(\operatorname{Inv}\left(N, f_{\lambda}\right)\right)$ does not depend on $\lambda \in \Lambda$ (for proof see $\left.\S 7\right)$.

Theorem 2.12 (additivity property). Assume an isolated invariant set $K$ is a disjoint sum of two other isolated invariant sets $K_{1}, K_{2}$. Then

$$
C(K)=C\left(K_{1}\right) \times C\left(K_{2}\right) .
$$

(For proof see $\S 7$.

\section{THE INDEX OF A HYPERBOLIC FIXED POINT AND A HYPERBOLIC PERIODIC ORBIT}

As we have seen, in case of the time-one map of a flow, the distinguished isomorphism of an index is always the identity. This is not true in general. The simplest example is a hyperbolic fixed point. 
Recall that a linear map $A: \mathbf{R}^{n} \rightarrow \mathbf{R}^{n}$ is called hyperbolic iff $A$ is an isomorphism and no eigenvalue of $A$ has modulus one. A point $x \in \mathbf{R}^{n}$ is called a hyperbolic fixed point of a $C^{1}$-diffeomorphism $f: \mathbf{R}^{n} \rightarrow \mathbf{R}^{n}$ iff $f(x)=x$ and $D f(x)$ is hyperbolic.

Let $x_{0}$ be a hyperbolic fixed point of $f$. Let $k$ denote the number of eigenvalues of $D f\left(x_{0}\right)$ with modulus greater than one (counted with multiplicity). Let $l$ denote the number of real eigenvalues of $D f\left(x_{0}\right)$ which are less than -1 . Then the pair $(k, l)$ will be called the Morse index of $x_{0}$.

Theorem 3.1. Assume $x_{0}$ is a hyperbolic fixed point of a $C^{1}$-diffeomorphism $f: \mathbf{R}^{n} \rightarrow \mathbf{R}^{n}$. Then $\left\{x_{0}\right\}$ is an isolated invariant set and

$$
C_{i}\left(\left\{x_{0}\right\}\right)= \begin{cases}0 & \text { for } i \neq k, \\ \left(\Xi,(-1)^{l} \text { id }\right) & \text { for } i=k,\end{cases}
$$

where $(k, l)$ is the Morse index of $\left\{x_{0}\right\}$.

The shape index of $\left\{x_{0}\right\}$ is the shape of the sphere of dimension $k$. Hence,, contrary to our index, it does not reflect whether the map is orientation preserving or reversing.

It is worth mentioning that the only feature of the Leray functor $L$ which is necessary to prove the above and the following theorems is the fact that $L$ restricted to $\mathscr{E} I$ is an identity. It is always so if we succeed in constructing an index pair $P$ with $I_{P}$ an isomorphism. However, one should note that there exist isolated invariant sets which do not admit such index pairs.

Proof of Theorem 3.1. Without loss of generality we can assume that $x_{0}=0$. Put $A:=D f(0)$.

First we shall consider the case when $f$ is linear. Then $A=f$ and $\mathbf{R}^{n}$ can be decomposed into two invariant subspaces on which either $A$ or $A^{-1}$ is expanding (cf. [Ni, Lemma 2.2]). Thus $\{0\}$ is the only compact trajectory of $f=A$ and consequently it is an isolated invariant set.

In order to prove (3.1), first assume that $A$ has $n$ different eigenvalues. Choose a basis in which $A$ has the block-diagonal matrix with one-dimensional blocks $\left[\lambda_{j}\right]$ corresponding to the real eigenvalues $\lambda_{j}$ and two-dimensional blocks

$$
\left[\begin{array}{cc}
r_{j} \cos \varphi_{j} & r_{j} \sin \varphi_{j} \\
-r_{j} \sin \varphi_{j} & r_{j} \cos \varphi_{j}
\end{array}\right]
$$

corresponding to the pairs of complex eigenvalues $r_{j} \exp \left(-i \varphi_{j}\right), r_{j} \exp \left(i \varphi_{j}\right)$. For $t \in[0,1]$ let $A_{t}$ be a map with the matrix of the same block-diagonal structure and with corresponding blocks of the form

$$
\left[h\left(\lambda_{j}, t\right)\right]
$$

and

$$
h\left(r_{j}, t\right)\left[\begin{array}{cc}
\cos t \varphi_{j} & \sin t \varphi_{j} \\
-\sin t \varphi_{j} & \cos t \varphi_{j}
\end{array}\right]
$$


where

$$
h(u, t):=t u+(1-t) \operatorname{sgn}(u) \exp (\operatorname{sgn}(|u|-1) \ln 2) .
$$

Then the origin is the only nontrivial isolated invariant set with respect to each $A_{t}$ and Theorem 2.11 shows that $C\left(\{0\}, A_{1}\right)=C\left(\{0\}, A_{0}\right)$. Thus it suffices to assume that $A=A_{0}$ has the diagonal matrix in which -2 appears $l$ times, 2 appears $k-l$ times, and the remaining nonzero entries are $\frac{1}{2}$ or $-\frac{1}{2}$.

Without loss of generality we can assume that $\mathbf{R}^{n}=\mathbf{R}^{k} \times \mathbf{R}^{n-k}$ is a decomposition of $\mathbf{R}^{n}$ into invariant subspaces corresponding to eigenvalues with modulus greater than one and less than one, respectively. Then $A=B \times C$, where $B: \mathbf{R}^{k} \rightarrow \mathbf{R}^{k}, C: \mathbf{R}^{n-k} \rightarrow \mathbf{R}^{n-k}$ are linear maps with diagonal matrices. In particular, we can assume that $B$ has the matrix

$$
\left[\begin{array}{cccccccc}
2 & 0 & \cdot & \cdot & \cdot & \cdot & \cdot & 0 \\
0 & 2 & & & & & & \cdot \\
\cdot & & \cdot & & & & & \cdot \\
\cdot & & & 2 & & & & \cdot \\
\cdot & & & & -2 & & & \cdot \\
\cdot & & & & & \cdot & & \cdot \\
\cdot & & & & & & -2 & 0 \\
0 & \cdot & \cdot & . & . & . & 0 & -2
\end{array}\right]
$$

with $(k-l)$ positive entries and $l$ negative entries.

Put

$$
\begin{aligned}
N & :=\left\{(x, y) \in \mathbf{R}^{k} \times \mathbf{R}^{n-k} \mid\|x\| \leq 2,\|y\| \leq 2\right\}, \\
P_{1} & :=\{(x, y) \in N \mid\|y\| \leq 1\}, \\
P_{2} & :=\left\{(x, y) \in P_{1} \mid\|x\| \geq 1\right\}, \\
B^{k} & :=\left\{x \in \mathbf{R}^{k} \mid\|x\| \leq 1\right\}, \\
S^{k-1} & :=\left\{x \in \mathbf{R}^{k} \mid\|x\|=1\right\} .
\end{aligned}
$$

It is easy to verify that $P:=\left(P_{1}, P_{2}\right)$ is an index pair of $\{0\}$.

Let the mappings

$$
\begin{aligned}
& d:\left(B^{k}, S^{k-1}\right) \rightarrow\left(B^{k}, S^{k-1}\right) \quad \text { and } \\
& \alpha:\left(B^{k}, S^{k-1}\right) \rightarrow\left(P_{1}, P_{2}\right)
\end{aligned}
$$

be given by

$$
\begin{aligned}
& d\left(x_{1}, x_{2}, \ldots, x_{k}\right):=\left(x_{1}, x_{2}, \ldots, x_{k-l},-x_{k-l+1}, \ldots,-x_{k}\right), \\
& \alpha(x):=(x, 0)
\end{aligned}
$$

From the homotopy commutative diagram

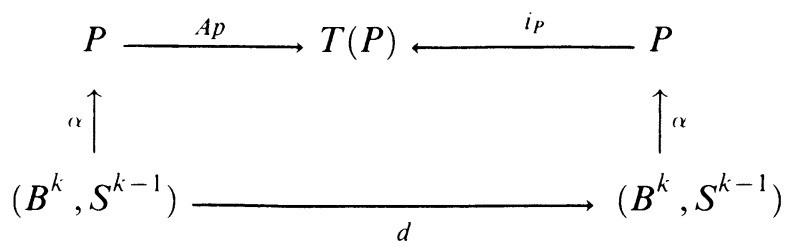


we get the following commutative diagram in cohomology:

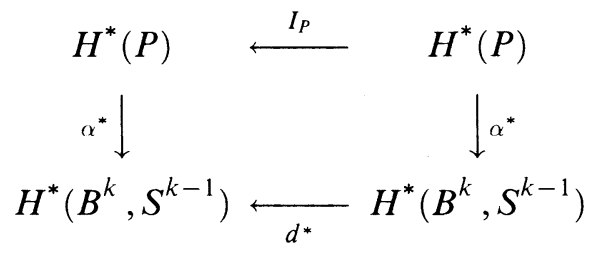

The homotopy and strong excision properties of the Alexander-Spanier cohomology imply that $\alpha^{*}$ is an isomorphism. Thus

$$
C(\{0\})=L\left(H^{*}(P), I_{P}\right)=L\left(H^{*}\left(B^{k}, S^{k-1}\right), d^{*}\right)=\left(H^{*}\left(B^{k}, S^{k-1}\right), d^{*}\right),
$$

because obviously $d^{*}$ is an isomorphism (cf. Proposition 4.1). It is now an elementary task of homology and cohomology theories to verify that $\left(H^{i}\left(B^{k}, S^{k-1}\right), d^{i}\right)$ is given by the right-hand side of formula (3.1).

In order to obtain that formula in case of a linear map with multiple eigenvalues it suffices to construct a homotopy joining the map $A$ with a close map $A^{\prime}$ which has pairwise different eigenvalues and then apply Theorem 2.11 and the case just proved.

Now we shall consider the case of a general $f$. The fact that also in this case $\{0\}$ is an isolated invariant set follows directly from the linear case and the Hartman-Grobman theorem (see [Ni, Theorem 2.2]).

We have

$$
f(x)=A x+r(x),
$$

where $r(x)=o(\|x\|)$. For $\lambda \in[0,1]$ define

$$
f_{\lambda}(x):=A x+\lambda r(x) .
$$

For each fixed $\lambda \in[0,1]$, we can apply the Hartman-Grobman theorem to find $\delta(\lambda)>0$ such that $B(\delta(\lambda)):=\left\{x \in \mathbf{R}^{n}:\|x\| \leq \delta(\lambda)\right\}$ is an isolating neighborhood with respect to $f_{\lambda}$, isolating $\{0\}$. A compactness argument together with Corollary 7.3 shows that there exists $\delta>0$ such that $B(\delta)$ is an isolating neighborhood with respect to $f_{\lambda}$ for each $\lambda \in[0,1]$. Obviously, we can make $\delta$ small enough to ensure that $\operatorname{Inv}(B(\delta), f)=\{0\}$. Since $f_{1}=f, f_{0}=A$, the thesis now follows from the homotopy invariance of the Conley index (Theorem 2.11) and the proved linear case.

Theorem 3.2. Let $x_{0} \in \mathbf{R}^{n}$ be a hyperbolic periodic orbit of $f$, i.e., a hyperbolic fixed point of $f^{d}$ for some $d \in \mathbf{N}$. Let $(k, l)$ be the Morse index of $x_{0}$ with respect to $f^{d}$. Assume $d$ is the minimal period of $x_{0}$, i.e., $f^{i}\left(x_{0}\right) \neq x_{0}$ for $i=$ $1,2, \ldots, d-1$. Then $K:=\left\{x_{0}, f\left(x_{0}\right), \ldots, f^{d-1}\left(x_{0}\right)\right\}$ is an isolated invariant set and

$$
C_{i}(K)= \begin{cases}0 & \text { for } i \neq k, \\ \left(\Xi^{d}, D\right) & \text { for } i=k,\end{cases}
$$


where $D: \Xi^{d} \rightarrow \Xi^{d}$ is given on the canonical basis $\left\{e_{i}\right\}_{i=1, d}$ of $\Xi^{d}$ by $D\left(e_{i}\right)=$ $e_{i+1}$ for $i=1,2, \ldots, d-1, D\left(e_{d}\right)=(-1)^{l} e_{1}$.

Proof. Choose $N$ an isolating neighborhood of $\left\{x_{0}\right\}$ with respect to $g:=$ $f^{d}$ and $P \in \operatorname{IP}(N, g)$. Put $x^{i}:=f^{i}\left(x_{0}\right), M^{i}:=f^{i}(N), Q^{i}:=f^{i}(P)$ for $i=0,1, \ldots, d-1$. Taking $N$ smaller, if necessary, we can assume that $M^{i} \cap M^{j}=\varnothing$ for $i \neq j$ and $f\left(M^{i}\right) \cap M^{j}=\varnothing$ for $j \neq(i+1) \bmod p$.

Obviously, $x^{i}$ are hyperbolic fixed points of $g$ with the same Morse index $(k, l)$. One can easily verify that $Q^{i} \in \operatorname{IP}\left(M^{i}, g\right)$ for $i=0,1, \ldots, d-1$. It follows that $M:=\bigcup M^{i}$ is an isolating neighborhood with respect to $f$, which isolates $\left\{x^{0}, x^{1}, \ldots, x^{d-1}\right\}$. Moreover, $Q:=\bigcup Q^{i} \in \operatorname{IP}(M, f)$. We have

$$
H^{*}(Q)=H^{*}\left(Q^{0}\right) \times \cdots \times H^{*}\left(Q^{d-1}\right) .
$$

Hence it follows from (3.1) that $H^{i}(Q)$ is zero for $i \neq k$ and $H^{k}(Q)$ is a $d$-dimensional free module.

Choose arbitrarily a generator $\alpha^{d-1} \in H^{k}\left(Q^{d-1}\right)$ and define recursively a sequence $\left\{\alpha^{i}\right\}_{i=0, d-1}$ of generators in $H^{k}\left(Q^{i}\right)$ by $\alpha^{i-1}:=H^{k}(f)\left(\alpha^{i}\right)$ for $i=$ $1,2, \ldots, d-1$. Then $\left\{\varepsilon^{i}\right\}_{i=0, d-1}$ with $\varepsilon^{i}:=\left(0, \ldots, \alpha^{i}, \ldots, 0\right)$ is a basis of $H^{k}(Q)$. It is straightforward to verify that $I_{Q, f}\left(\varepsilon^{i}\right)=\varepsilon^{i-1}$ for $i=1,2, \ldots, d-$ 1 . We also have by (3.1) that

$$
\begin{aligned}
I_{Q, f}\left(\varepsilon^{0}\right) & =I_{Q, f^{d}}\left(\varepsilon^{d-1}\right)=\left(0, \ldots, 0, I_{P, g}\left(\alpha^{d-1}\right)\right) \\
& =\left(0, \ldots, 0,(-1)^{l} \alpha^{d-1}\right)=(-1)^{1} \varepsilon^{d-1} .
\end{aligned}
$$

This shows that $I_{Q, f}$ is an isomorphism, hence $\left(H^{*}(Q), I_{Q, f}\right)=C(K)$ is of the form (3.2).

\section{THE LERAY FUNCTOR}

In this section we construct the Leray functor and prove its main properties.

Let $(F, f) \in \mathscr{E} E$. Define the generalized kernel of $f$ as

$$
\operatorname{gker}(f):=\bigcup\left\{f^{-n}(0) \mid n \in \mathbf{N}\right\} .
$$

Since $f(\operatorname{gker}(f)) \subseteq \operatorname{gker}(f)$, we have an induced monomorphism

$$
f^{\prime}: F / \operatorname{gker}(f) \ni[x] \rightarrow[f(x)] \in F / \operatorname{gker}(f) .
$$

Put

$$
L M(F, f):=\left(F / \operatorname{gker}(f), f^{\prime}\right) \in \mathscr{E} M .
$$

Assume $\varphi:(E, e) \rightarrow(F, f)$ is a morphism. The commutativity of the diagram (1.1) implies that $\varphi(\operatorname{gker}(e)) \subseteq \operatorname{gker}(f)$; thus we have an induced mapping 
$\varphi^{\prime}: E / \operatorname{gker}(e) \rightarrow F / \operatorname{gker}(f)$. Obviously, the diagram

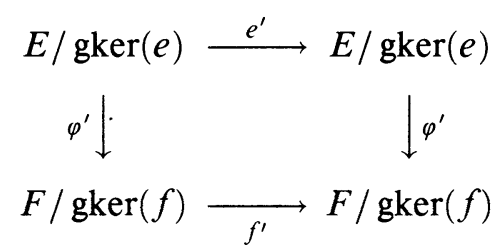

commutes. We put

$$
L M(\varphi):=\varphi^{\prime} .
$$

It is straightforward to verify that (4.1) and (4.2) define a covariant functor

$$
L M: \mathscr{E} E \rightarrow \mathscr{E} M .
$$

Now assume that $(F, f) \in \mathscr{E} M$. Define the generalized image of $f$ as

$$
\operatorname{gim}(f):=\bigcap\left\{f^{n}(F) \mid n \in \mathbf{N}\right\} .
$$

Since $f(\operatorname{gim}(f)) \subseteq \operatorname{gim}(f)$, we can consider the contraction

$$
f^{\prime \prime}: \operatorname{gim}(f) \ni x \rightarrow f(x) \in \operatorname{gim}(f) .
$$

Obviously, $f^{\prime \prime}$ is a monomorphism, as a contraction of a monomorphism. To see that $f^{\prime \prime}$ is also an epimorphism, take $y \in \operatorname{gim}(f)$. Then there exists a sequence $\left\{x_{n} \mid n \in \mathbf{N}\right\} \subseteq F$ such that $f^{n}\left(x_{n}\right)=y$ for all $n \in \mathbf{N}$. Since $f$ is a monomorphism, we get $f\left(x_{n+1}\right)=x_{n}$ and $f^{n}\left(x_{n+1}\right)=x_{1}$ for all $n \in \mathbf{N}$. Thus $x_{1} \in \operatorname{gim}(f)$ and $y=f\left(x_{1}\right) \in f(\operatorname{gim}(f))$. This shows that $f^{\prime \prime}$ is an isomorphism. Put

$$
L I(F, f):=\left(\operatorname{gim}(f), f^{\prime \prime}\right) \in \mathscr{E} I .
$$

Assume $(E, e),(F, f) \in \mathscr{E} M$ and $\varphi:(E, e) \rightarrow(F, f)$. It follows from the commutativity of the diagram $(1.1)$ that $\varphi(\operatorname{gim}(e)) \subseteq \operatorname{gim}(f)$. Let $\varphi^{\prime \prime}$ denote the contraction $\varphi^{\prime \prime}: \operatorname{gim}(e) \ni x \rightarrow \varphi(x) \in \operatorname{gim}(f)$. We then have the commutative diagram

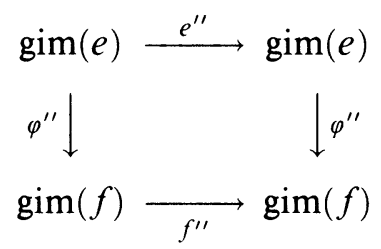

which shows that (4.3) together with

$$
L I(\varphi):=\varphi^{\prime \prime}
$$

defines a covariant functor

$$
L I: \mathscr{E} M \rightarrow \mathscr{E} I
$$

The functor

$$
L: \mathscr{E} E \rightarrow \mathscr{E} I
$$

defined as the composition $L I \circ L M$ will be called the Leray functor.

Note the following obvious proposition. 
Proposition 4.1. If $(E, e) \in \mathscr{E} I$ then $L(E, e)=(E, e)$. Moreover, if also $(F, f) \in \mathscr{E} I$ and $\varphi:(E, e) \rightarrow(F, f)$ then $L(\varphi)=\varphi$. (Identities here are understood as natural, obvious isomorphisms.)

Observe that if $(E, e) \in \mathscr{E} E$ then $e$ can be considered as a morphism $e:(E, e) \rightarrow(E, e)$, because obviously the diagram

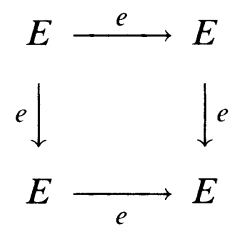

commutes. In particular, $L(e)$ makes sense and it can be understood both as a morphism in $\mathscr{E} I$ and as the endomorphism with which $L(E, e)$ is equipped.

Remark 4.2. If $E \in \mathscr{E}$ and $e \in \mathscr{E}(E, E)$ is an isomorphism then $e:(E, e) \rightarrow$ $(E, e)$ is an isomorphism in $\mathscr{E} I$.

Definition 4.3. We say that two objects $(E, e),(F, f) \in \mathscr{E} E$ are linked by $\varphi \in \mathscr{E}(E, F), \psi \in \mathscr{E}(F, E)$ iff the following diagram commutes

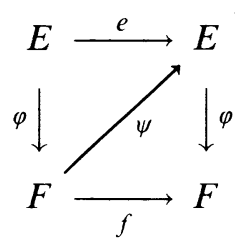

Note that the commutativity of diagram (4.4) in particular means that $\varphi:(E, e)$ $\rightarrow(F, f), \psi:(F, f) \rightarrow(E, e)$, and the following diagram commutes.

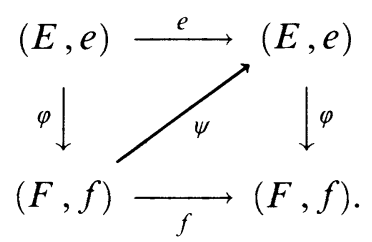

Applying the Leray functor to the above diagram, we get the following commutative diagram in $\mathscr{E} I$ :

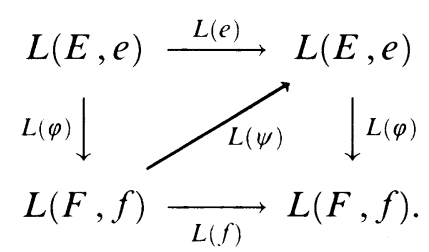

Since $L(e)$ and $L(f)$ are isomorphisms in $\mathscr{E}$ and consequently also in $\mathscr{E} I$, we see that $L(\varphi)$ and $L(\psi)$ are also isomorphisms. This proves the following theorem, which allows us to overcome the lack of the trajectory-defined homotopy in the construction of the Conley index for the discrete case. 
Theorem 4.4. Assume $(E, e)$ and $(F, f)$ are linked by $\varphi \in \mathscr{E}(E, F), \psi \in$ $\mathscr{E}(F, E)$. Then $L(\varphi), L(\psi)$ are isomorphisms; in particular, $L(E, e)$ and $L(F, f)$ are isomorphic.

We finish this section with the following two simple propositions.

Proposition 4.5. The product of $(E, e),(F, f) \in \mathscr{E} E(\mathscr{E} I)$ in the category $\mathscr{E} E$ $(\mathscr{E} I)$ is given by

$$
(E, e) \times(F, f):=(E \times F, e \times f) .
$$

Moreover,

$$
L((E, e) \times(F, f))=L(E, e) \times L(F, f) .
$$

Proposition 4.6. $L(0)=0$.

\section{CONSTRUCTION OF INDEX PAIRS FOR THE DISCRETE CASE}

We begin with the following

\section{Proposition 5.1.}

(5.1) $A \subseteq B \Rightarrow \operatorname{Inv} A \subseteq \operatorname{Inv} B, \operatorname{Inv}^{+} A \subseteq \operatorname{Inv}^{+} B, \operatorname{Inv}^{-} A \subseteq \operatorname{Inv}^{-} B$;

(5.2) $\operatorname{Inv}(\operatorname{Inv} A)=\operatorname{Inv} A, \operatorname{Inv}^{+}\left(\operatorname{Inv}^{+} A\right)=\operatorname{Inv}^{+} A, \operatorname{Inv}^{-}\left(\operatorname{Inv}^{-} A\right)=\operatorname{Inv}^{-} A$;

(5.3) $\operatorname{Inv} A=\operatorname{Inv}^{+} A \cap \operatorname{Inv}^{-} A$.

As a consequence of (5.2) we get

Proposition 5.2. For every $A \subseteq X$ Inv $A$ is invariant. It is the maximal invariant subset of $A$.

Proposition 5.3. If $N$ is compact then $\operatorname{Inv}^{+} N, \operatorname{Inv}^{-} N, \operatorname{Inv} N$ are compact.

Lemma 5.4. Assume $M$ is compact and for every $n \in \mathbf{N}$ there exists $x_{n} \in M$ such that $f^{i}\left(x_{n}\right) \in M$ for all $i=0,1,2, \ldots, n$. Then $\operatorname{Inv} M \neq \varnothing$.

Proof. Put $y_{n}=f^{n}\left(x_{2 n}\right)$. Then $f^{i}\left(y_{n}\right) \in M$ for $i \in\{-n,-n+1, \ldots, n-1, n\}$. Without loss of generality we can assume that $y_{n} \rightarrow y \in M$. Fix $k \in \mathbf{Z}$. Since for almost all $n \in \mathbf{N}$ we have $f^{k}\left(y_{n}\right) \in M$; there is also $f^{k}(y) \in M$. Thus $y \in \operatorname{Inv} M$.

For $x \in N$ put

$$
\begin{aligned}
& \alpha_{N}(x):=\sup \left\{n \in \mathbf{Z}^{+} \mid f^{-i}(x) \in N \text { for } i=0,1, \ldots, n\right\}, \\
& \omega_{N}(x):=\sup \left\{n \in \mathbf{Z}^{+} \mid f^{i}(x) \in N \text { for } i=0,1, \ldots, n\right\} .
\end{aligned}
$$

We shall need the following m.v. maps

$$
F d_{N}:=F d_{N, f}: N \ni x \rightarrow\left\{f^{i}(x) \mid i=0,1, \ldots, \omega_{N}(x)\right\} \subseteq N
$$

and

$$
B k_{N}:=B k_{N, f}: N \ni x \rightarrow\left\{f^{-i}(x) \mid i=0,1, \ldots, \alpha_{N}(x)\right\} \subseteq N
$$


Lemma 5.5. The m.v. mappings $F d_{N}, B k_{N}$ are u.s.c. on sets $N \backslash \operatorname{Inv}^{+} N$, $N \backslash \operatorname{Inv}^{-} N$, respectively.

Proof. Consider $F d_{N}$. Let $x \in N \backslash \operatorname{Inv}^{+} N$ and $U \supseteq F d_{N}(x)$ be open. Put $n:=\omega_{N}(x)$. Then $f^{n+1}(x) \notin N$. Choose $V$ a neighborhood of $x$ so that $f^{i}(v) \subseteq U$ for $i=0,1, \ldots, n$ and $f^{n+1}(v) \subseteq X \backslash N$. Then $F d_{N}(V) \subseteq$ $\bigcup\left\{f^{i}(V) \mid i=0,1, \ldots, n\right\} \subseteq U$.

The case of $B k_{N}$ is similar.

Lemma 5.6. Assume $Z$ is compact and $Z \cap \operatorname{Inv}^{+} N=\varnothing$. Then $F d_{N}(Z)$ is compact and

$$
F d_{N}(Z) \cap \operatorname{Inv}^{+} N=\varnothing,
$$

i.e., $N \backslash F d_{N}(Z)$ is a neighborhood of $\operatorname{Inv}^{+} N$.

Proof. Compactness of $F d_{N}(Z)$ follows directly from the above lemma and Proposition 1.1. The remaining assertion is obvious.

Lemma 5.7. Assume $Z$ is a compact neighborhood of $\operatorname{Inv}^{-} N$ in $N$. Then $F d_{N}(Z)$ is compact.

Proof. It suffices to show that $F d_{N}(Z)$ is closed. Let $\left\{y_{n}\right\} \subseteq F d_{N}(Z)$ be a sequence converging to $y \in N$. If $y \in \operatorname{Inv}^{-} N$ then $y \in Z \subseteq F d_{N}(Z)$. Thus assume $y \notin \operatorname{Inv}^{-} N$, i.e., $f^{-k}(y) \notin N$ for some $k \in \mathbf{N}$. Since $y_{n} \in F d_{N}(Z)$, one can find sequences $\left\{j_{n}\right\} \subseteq \mathbf{Z}^{+},\left\{z_{n}\right\} \subseteq Z$ such that $f^{j_{n}}\left(z_{n}\right)=y_{n}, j_{n} \in$ $\left\{0,1, \ldots, \omega_{N}\left(z_{n}\right)\right\}$. Since $f^{-k}(w) \notin N$ for $w$ sufficiently close to $y$, we see that the sequence $\left\{j_{n}\right\}$ is bounded. Taking subsequences, if necessary, we can assume that $j_{n} \rightarrow j \in \mathbf{Z}^{+}, z_{n} \rightarrow z \in Z$. Thus $f^{j}(z)=y$ and $f^{i}(z) \in N$ for $i=0,1, \ldots, j$, which means that $y \in F d_{N}(Z)$.

Lemma 5.8. Assume $A$ is compact, positively invariant with respect to $N$, and Inv $^{-} N \subseteq A$. Then for every open neighborhood $V$ of $A$ there exists a compact neighborhood $Z$ of $A$ in $N$ such that

$$
F d_{N}(Z) \subseteq V
$$

Proof. Compactness of $N \backslash V$ implies that there exists $k \in \mathbf{N}$ such that $f^{-k}(y)$ $\notin N$ for all $y \in N \backslash V$. Let $x \in A$ and

$$
n(x):=\min \left(k, \omega_{N}(x)\right) .
$$

Obviously

$$
f^{j}(x) \in A \subseteq V \quad \text { for all } j=0,1, \ldots, n(x) .
$$

Thus one can find for every $x \in A$ a compact neighborhood $W_{x}$ of $x$ in $N$ such that

$$
f^{j}\left(W_{x}\right) \subseteq V \quad \text { for } j=0,1, \ldots, n(x) .
$$

Taking a smaller neighborhood, if necessary, we can also ensure that $n(z) \leq$ $n(x)$ for all $z \in W_{x}$. By compactness of $A$ there exists a finite $A_{0} \subseteq A$ such 
that $A \subseteq Z:=\bigcup\left\{W_{x} \mid x \in A_{0}\right\}$. Then

$$
f^{j}(z) \in V \quad \text { for all } z \in Z \text { and } j=0,1, \ldots, n(z) .
$$

Obviously $Z$ is a neighborhood of $A$. In order to show that such $Z$ satisfies (5.4) take $x \in F d_{N}(Z)$. Then there exist $z \in Z$ and $m \in \mathbf{Z}^{+}$such that $x=f^{m}(z)$ and $m=\omega_{N}(z)$. If $m \leq k$, then $m \leq n(z)$ and $x \in V$ by (5.5). Thus assume $m>k$. If $x \notin V$, then $f^{-k}(x) \notin N$, which contradicts $f^{-k}(x)=f^{m-k}(z) \in N$. Thus $x \in V$. This finishes the proof.

Lemma 5.9. Assume $U$ and $V$ are open neighborhoods of $\operatorname{Inv}^{+} N$ and $\operatorname{Inv}^{-} N$, respectively. Then there exists an index pair $P$ in $N$ such that

$$
P_{1} \subseteq V, \quad N \backslash P_{2} \subseteq U
$$

Proof. Without loss of generality we can assume that

$$
U \cap V \subseteq \operatorname{int} N \cap f^{-1}(\text { int } N) \text {. }
$$

Using Lemma 5.8 choose a compact neighborhood $Z$ of $A:=\operatorname{Inv}^{-} N$ such that $F d_{N}(Z) \subseteq V$. Put $P_{1}:=F d_{N}(Z)$ and $P_{2}:=F d_{N}(N \backslash U)$. Then by Lemmas 5.6 and 5.7, $P_{1}, P_{2}$ are compact and obviously they are positively invariant with respect to $N$. There is also $\operatorname{Inv}^{-} N \subseteq$ int $_{N} Z \subseteq$ int $_{N} P_{1} \subseteq P_{1} \subseteq V$. Since $(N \backslash U) \cap \operatorname{Inv}^{+} N=\varnothing$, we get from Lemma 5.6 that $F d_{N}(N \backslash U) \cap \operatorname{Inv}^{+} N=\varnothing$. Hence $\operatorname{Inv}^{+} N \subseteq N \backslash P_{2}$. There is also $N \backslash U \subseteq F d_{N}(N \backslash U)=P_{2}$, thus $N \backslash P_{2} \subseteq$ $U$. Finally,

$$
P_{1} \backslash P_{2} \subseteq U \cap V \subseteq \text { int } N \cap f^{-1}(\text { int } N),
$$

which shows that $P:=\left(P_{1}, P_{2}\right) \in \operatorname{IP}(N)$.

We are now able to prove Theorem 2.3 on the existence of index pairs.

Proof of Theorem 2.3. Choose neighborhoods $U$ of $\operatorname{Inv}^{+} N$ and $V$ of $\operatorname{Inv}^{-} N$ in $N$ such that $U \cap V \subseteq W$. Then by Lemma 5.9 there exists an index pair $P$ in $N$ such that (5.6) is satisfied. Thus we have $P_{1} \backslash P_{2} \subseteq U \cap V \subseteq W$.

We shall need some more lemmas on the existence of index pairs. We begin with a definition which is important in the construction of the cohomological index.

Definition 5.10. Assume $P, Q \in \operatorname{IP}(N)$. We shall say that $P$ is related to $Q$ iff $P \subseteq Q$ and

$$
\operatorname{cl}\left(Q_{1} \backslash P_{2}\right) \subseteq \text { int } N \cap f^{-1}(\text { int } N) .
$$

Remark 5.11. If $P$ is related to $Q$ then $\left(Q_{1}, P_{2}\right) \in \operatorname{IP}(N)$.

Lemma 5.12. For every isolating neighborhood $N$ there exist $P, Q \in \operatorname{IP}(N)$ such that $P \subseteq$ int $_{N} Q$ and $P$ is related to $Q$.

Proof. Let $W$ be a neighborhood of Inv $N$ such that

$$
\text { cl } W \subseteq \text { int } N \cap f^{-1}(\text { int } N) \text {. }
$$


By Theorem 2.3 we can choose $Q_{1}$ and $P_{2}$ such that $\left(Q_{1}, P_{2}\right) \in \operatorname{IP}(N)$ and $Q_{1} \backslash P_{2} \subseteq W$. Then also

$$
\operatorname{cl}\left(Q_{1} \backslash P_{2}\right) \subseteq \operatorname{cl} W \subseteq \operatorname{int} N \cap f^{-1}(\text { int } N) .
$$

Select $U^{\prime}$ open in $N$ such that

$$
P_{2} \subseteq U^{\prime} \subseteq \operatorname{cl} U^{\prime} \subseteq N \backslash \operatorname{Inv}^{+} N
$$

Using Lemma 5.9 we can find $\left(P_{1}, Q_{2}\right) \in \operatorname{IP}(N)$ such that $Q_{1} \subseteq \operatorname{int}_{N} P_{1}$, $N \backslash Q_{2} \subseteq U:=N \backslash \operatorname{cl} U^{\prime}$. Then

$$
P_{2} \subseteq U^{\prime} \subseteq \mathrm{cl} U^{\prime}=N \backslash U \subseteq Q_{2},
$$

hence $P_{2} \subseteq$ int $_{N} Q_{2}$. Put $P:=\left(P_{1}, P_{2}\right)$ and $Q:=\left(Q_{1}, Q_{2}\right)$. We have just shown that $P \subseteq$ int $_{N} Q$. There are also

$$
\begin{gathered}
P_{1} \backslash P_{2} \subseteq Q_{1} \backslash P_{2} \subseteq \text { int } N \cap f^{-1}(\text { int } N), \\
Q_{1} \backslash Q_{2} \subseteq Q_{1} \backslash P_{2} \subseteq \text { int } N \cap f^{-1}(\text { int } N),
\end{gathered}
$$

hence $P, Q \in \operatorname{IP}(N)$. Finally (5.7) implies that $P$ is related to $Q$.

Proposition 5.13. Assume $A$ is compact and positively invariant with respect to $N, A \cap \operatorname{Inv}^{+} N=\varnothing$, and $A \subseteq U$ for some $U$ open in $N$. Then there exists a compact neighborhood $Z$ of $A$ in $N$ such that $F d_{N} Z \subseteq U$.

Proof. Positive invariance of $A$ with respect to $N$ implies that $F d_{N} A \subseteq A \subseteq$ $U$. Using the upper semicontinuity of $F d_{N}$ we can find for every $x \in A$ a compact neighborhood $V_{x}$ in $N$ such that $F d_{N}\left(V_{x}\right) \subseteq U$. Choose a finite subset $A_{0} \subseteq A$ such that $Z:=\bigcup\left\{V_{x} \mid x \in A_{0}\right\} \supseteq A$. Then $Z$ is compact and $F d_{N}(Z) \subseteq U$.

Proposition 5.14. Assume $P, Q, R \in \operatorname{IP}(N), P \subseteq Q \subseteq R$. If $P, R$ are related then so are $P, Q$ and $Q, R$.

Lemma 5.15. Assume $P, R \in \operatorname{IP}(N), P, R$ are related, and $P \subseteq$ int $_{N} R$. Then there exists $Q \in \operatorname{IP}(N)$ such that $P \subseteq \operatorname{int}_{N} Q, Q \subseteq \operatorname{int}_{N} R, P$ is related to $Q$, $Q$ is related to $R$.

Proof. Using Proposition 5.13 and Lemma 5.8 for $i=1,2$ find a compact neighborhood $A_{i}$ of $P_{i}$ such that $F d_{N}\left(A_{i}\right) \subseteq$ int $_{N} R_{i}$. Put $Q_{i}:=F d_{N} A_{i} \cdot Q_{1}$, $Q_{2}$ are obviously positively invariant with respect to $N$. There are

$$
\begin{aligned}
& \operatorname{Inv}^{-} N \subseteq \operatorname{int} P_{1} \subseteq \operatorname{int}_{N} A_{1} \subseteq \operatorname{int}_{N} Q_{1}, \\
& \operatorname{Inv}^{+} N \subseteq N \backslash R_{2} \subseteq N \backslash Q_{2} \subseteq N \backslash A_{2} .
\end{aligned}
$$

Thus (2.2) is satisfied and $Q_{1}, Q_{2}$ are compact by Lemmas 5.6, 5.7.

We have also

$$
Q_{1} \backslash Q_{2} \subseteq R_{1} \backslash P_{2} \subseteq \text { int } N \cap f^{-1}(\text { int } N) .
$$

Thus $Q:=\left(Q_{1}, Q_{2}\right) \in \operatorname{IP}(N) . \quad P \subseteq$ int $_{N} Q, Q \subseteq$ int $_{N} R$ are obvious. The remaining part of the lemma follows from Proposition 5.14. 


\section{Construction of the COHOMOlogical CONLEY INDEX}

Assume $A \subseteq B \subseteq N$ are compact and $A, B$ are positively invariant with respect to $N$. Define the set

$$
G(B):=G_{A, N}(B):=A \cup(f(B) \cap N) .
$$

The properties of the set $G(B)$ are summarized in the following easy

\section{Proposition 6.1.}

(6.1) $G(B)$ is compact and positively invariant with respect to $N$,

(6.2) $A \subseteq G(B) \subseteq B$,

(6.3) $f(B) \cap N \subseteq G(B)$.

Note that by (6.1) $G=G_{A, N}$ can be considered as a map from the family of all compact subsets of $N$, positively invariant with respect to $N$ into itself. In particular, for all $n \in \mathbf{N} G^{n}(B)$, the $n$th iterate of $G$ at $B$ is defined.

Lemma 6.2. Assume $\operatorname{Inv} \operatorname{cl}(B \backslash A)=\varnothing$. Then there exists $n \in \mathbf{N}$ such that $G^{n}(B)=A$.

Proof. Assume the contrary. Then $A \neq G^{n}(B)$ for all $n \in \mathbf{N}$. However, $A \subseteq G^{n}(B)$; thus there exists a sequence $\left\{y_{n}\right\} \subseteq N$ such that $y_{n} \in G^{n}(B) \backslash A$. This means that for every $n \in \mathbf{N}$ there exists $x_{n} \in B \backslash A \subseteq \operatorname{cl}(B \backslash A)$ such that $f^{i}\left(x_{n}\right) \in B \backslash A \subseteq \operatorname{cl}(B \backslash A)$ for $i=0,1, \ldots, n$. It follows now from Lemma 5.4 that $\operatorname{Inv} \operatorname{cl}(B \backslash A) \neq \varnothing$, a contradiction.

Assume $P, Q \in \operatorname{IP}(N), P \subseteq Q$. Put

$$
G(Q):=G_{P, N}(Q):=\left(G_{P_{1}, N}\left(Q_{1}\right), G_{P_{2}, N}\left(Q_{2}\right)\right) .
$$

Lemma 6.3. If $P, Q \in \operatorname{IP}(N), P$ is related to $Q$, then

(6.4) $G(Q) \in \operatorname{IP}(N)$,

(6.5) $P$ is related to $G(Q), G(Q)$ is related to $Q$,

(6.6) $f(Q) \cap N \subseteq G(Q)$.

Proof. Let $R_{i}:=G_{P_{i}, N}\left(Q_{i}\right)$ for $i=1,2$. It follows from Proposition 6.1 that $R_{1}, R_{2}$ are compact, positively invariant with respect to $N$, and $P \subseteq R \subseteq$ $Q$. Thus Inv $^{-} N \subseteq \operatorname{int}_{N} P_{1} \subseteq$ int $_{N} R_{1}$, Inv $^{+} N \subseteq N \backslash Q_{2} \subseteq N \backslash R_{2}, R_{1} \backslash R_{2} \subseteq$ $Q_{1} \backslash P_{2} \subseteq$ int $N \cap f^{-1}$ (int $\left.N\right)$. The last inclusion follows from Remark 5.11. This proves that $R \in \operatorname{IP}(N)$. We also have that

$$
\begin{aligned}
& \operatorname{cl}\left(R_{1} \backslash P_{2}\right) \subseteq \operatorname{cl}\left(Q_{1} \backslash P_{2}\right) \subseteq \operatorname{int} N \cap f^{-1}(\operatorname{int} N), \\
& \operatorname{cl}\left(Q_{1} \backslash R_{2}\right) \subseteq \operatorname{cl}\left(Q_{1} \backslash P_{2}\right) \subseteq \operatorname{int} N \cap f^{-1}(\operatorname{int} N),
\end{aligned}
$$

which proves (6.5). Property (6.6) follows from (6.3).

Theorem 6.4. Assume $f: X \rightarrow X$ is a homeomorphism, $N$ is an isolating neighborhood with respect to $f$, and $P, Q \in \operatorname{IP}(N), P \subseteq Q$. Then the inclusion

$$
\iota:\left(P_{1}, P_{1} \cap P_{2}\right) \rightarrow\left(Q_{1}, Q_{1} \cap Q_{2}\right)
$$


induces an isomorphism $L H^{*}(l):=L\left(H^{*}(l)\right): L\left(H^{*}(Q), I_{Q}\right) \rightarrow L\left(H^{*}(P), I_{P}\right)$. Proof. The proof consists of four steps.

Step 1. In this step we make the following three additional assumptions:

(6.7) $P_{2} \subseteq P_{1}, Q_{2} \subseteq Q_{1}$,

(6.8) $P$ is related to $Q$,

(6.9) $f(Q) \subseteq T_{N}(P)$.

Condition (6.9) enables us to define the mapping

$$
f_{Q P}: Q \ni x \rightarrow f(x) \in T_{N}(P)
$$

and

$$
I_{Q P}:=H^{*}\left(f_{Q P}\right) \circ H^{*}\left(i_{P}\right)^{-1} .
$$

We have the following commutative diagram, in which vertical arrows denote inclusions:

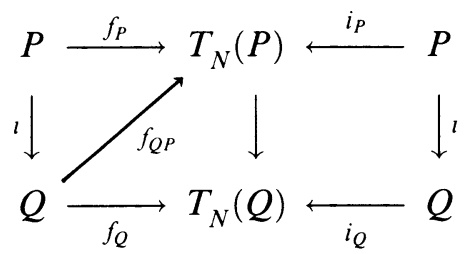

Applying $H^{*}$ to the above diagram and removing $H^{*}\left(T_{N}(P)\right), H^{*}\left(T_{N}(Q)\right)$, we obtain the following commutative diagram:

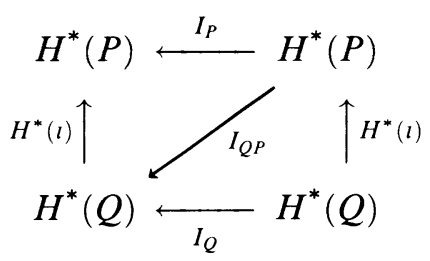

which shows that $\left(H^{*}(P), I_{P}\right)$ and $\left(H^{*}(Q), I_{Q}\right)$ are linked. Theorem 4.4 then shows that $L H^{*}(l)$ is an isomorphism.

Step 2. Now we assume that only (6.7) and (6.8) are satisfied. We see from Proposition 5.1 that

$$
\operatorname{Inv} \operatorname{cl}\left(Q_{1} \backslash P_{1}\right) \subseteq \operatorname{cl}\left(Q_{1} \backslash P_{1}\right) \cap \operatorname{Inv} N \subseteq \operatorname{cl}\left(N \backslash P_{1}\right) \cap \operatorname{int}_{N} P_{1}=\varnothing ;
$$

thus $\operatorname{Inv}\left(Q_{1} \backslash P_{1}\right)=\varnothing$. Similarly,

$$
\operatorname{Inv} \operatorname{cl}\left(Q_{2} \backslash P_{2}\right) \subseteq Q_{2} \cap \operatorname{Inv} N \subseteq Q_{2} \cap\left(N \backslash Q_{2}\right)=\varnothing ;
$$

i.e., also $\operatorname{Inv} \operatorname{cl}\left(Q_{2} \backslash P_{2}\right)=\varnothing$.

Thus we can apply Lemma 6.2 to find $n \in \mathbf{N}$ such that $G^{n}(Q)=P$. Put $Q^{i}:=G^{i}(Q)$. Then an induction argument based on Lemma 6.3 shows that $\left\{Q^{i}\right\}_{i=0, n}$ is a sequence of index pairs in $N$ such that $Q^{0}=Q, Q^{n}=P$, and $Q^{i+1}$ is related to $Q^{i}, f\left(Q^{i}\right) \cap N \subseteq Q^{i+1}$. Let $l_{i}: Q^{i+1} \rightarrow Q^{i}$ denote inclusion. It follows from Step 1 applied to index pairs $Q^{i+1}, Q^{i}$ that 
$L H^{*}\left(l_{i}\right): L H^{*}\left(Q^{i}\right) \rightarrow L H^{*}\left(Q^{i+1}\right)$ is an isomorphism and consequently also $L H^{*}(l)$ is an isomorphism as a superposition of isomorphisms $L H^{*}\left(l_{i}\right)$. This finishes the proof of Step 2.

Step 3. Now we assume that the only additional assumption is (6.7). Put $R_{1}:=$ $P_{1} \cup Q_{2}, R_{2}:=P_{1} \cap Q_{2}$. Then $R_{1}, R_{2}$ are compact, positively invariant with respect to $N$, and

$$
\operatorname{Inv}^{-} N \subseteq \operatorname{int}_{N} P_{1} \subseteq \operatorname{int}_{N} R_{1}, \quad \operatorname{Inv}^{+} N \subseteq N \backslash Q_{2} \subseteq N \backslash R_{2} .
$$

We also have

$$
\begin{aligned}
& P_{1} \backslash R_{2} \subseteq P_{1} \backslash P_{2} \subseteq \text { int } N \cap f^{-1}(\text { int } N), \\
& R_{1} \backslash Q_{2} \subseteq Q_{1} \backslash Q_{2} \subseteq \text { int } N \cap f^{-1}(\text { int } N) .
\end{aligned}
$$

This shows that $\left(P_{1}, R_{2}\right),\left(R_{1}, Q_{2}\right) \in \operatorname{IP}(N)$. (Note that $\left(R_{1}, R_{2}\right)$ need not be an index pair.) Consider the following commutative diagram of inclusions:

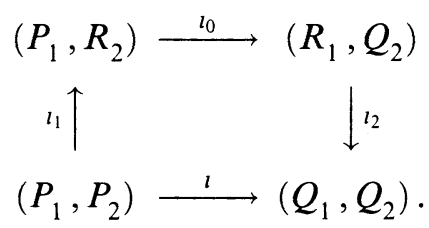

Since $P_{1} \backslash R_{2}=P_{1} \backslash Q_{2}=R_{1} \backslash Q_{2}$, it follows from the strong excision property of the Alexander-Spanier cohomology that $l_{0}^{*}$ is an isomorphism; hence $L H^{*}\left(l_{0}\right)$ is also an isomorphism. It is straightforward that $\left(P_{1}, P_{2}\right)$ is related to $\left(P_{1}, R_{2}\right)$ and $\left(R_{1}, Q_{2}\right)$ is related to $\left(Q_{1}, Q_{2}\right)$. Thus we can apply Step 2 to conclude that $L H^{*}\left(l_{1}\right)$ and $L H^{*}\left(l_{2}\right)$ are also isomorphisms. Hence

$$
L H^{*}(l)=L H^{*}\left(l_{1}\right) \circ L H^{*}\left(l_{0}\right) \circ L H^{*}\left(l_{2}\right)
$$

is an isomorphism, which finishes the proof of Step 3.

Step 4. No additional assumptions. Put $Q_{2}^{\prime}:=Q_{2} \cap Q_{1}, P_{2}^{\prime}:=P_{2} \cap P_{1}$. It is obvious that $P^{\prime}:=\left(P_{1}, P_{2}^{\prime}\right), Q^{\prime}:=\left(Q_{1}, Q_{2}^{\prime}\right)$ are index pairs in $N$. Thus the assertion of the theorem follows directly from Step 3 applied to $P^{\prime}$ and $Q^{\prime}$.

Corollary 6.5. For any $P, Q \in \operatorname{IP}(N), L\left(H^{*}(P), I_{P}\right)$ and $L\left(H^{*}(Q), I_{Q}\right)$ are isomorphic.

Proof. Let $R:=P \cap Q \in \operatorname{IP}(N)$. Since $R \subseteq P$ and $R \subseteq Q$, the above theorem implies that $L\left(H^{*}(P), I_{P}\right)=L\left(H^{*}(R), I_{R}\right)=L\left(H^{*}(Q), I_{Q}\right)$.

Proof of Theorem 2.6. First consider the case $M \subseteq N$. It follows from Corollary 6.5 that it suffices to show only the existence of index pairs $P \in \operatorname{IP}(N)$ and $Q \in \operatorname{IP}(M)$ such that $L\left(H^{*}(P), I_{P}\right)$ and $L\left(H^{*}(Q), I_{Q}\right)$ are isomorphic. To this end choose $P \in \operatorname{IP}(N)$ such that $P_{1} \backslash P_{2} \subseteq$ int $M \cap f^{-1}$ (int $M$ ) (the existence of such a pair is implied by Theorem 2.3) and put $Q_{i}:=M \cap P_{i}$ for $i=1,2$. One can easily verify that $Q:=\left(Q_{1}, Q_{2}\right) \in \operatorname{IP}(M)$. We also have 
$Q_{1} \backslash Q_{2}=M \cap\left(P_{1} \backslash P_{2}\right)=P_{1} \backslash P_{2}$; thus the inclusion $Q \subseteq P$ induces an isomorphism of $H^{*}(Q)$ and $H^{*}(P)$ by the strong excision property of the AlexanderSpanier cohomology, which shows that $L\left(H^{*}(P), I_{P}\right)$ and $L\left(H^{*}(Q), I_{Q}\right)$ are also isomorphic.

If $M, N$ are arbitrary isolating neighborhoods isolating $K$ then the assertion of the theorem follows from the just proved case applied to isolating neighborhoods $M \cap N, N$ and $M \cap N, M$.

\section{PROOFS OF HOMOTOPY AND ADDITIVITY PROPERTIES}

Assume $\Lambda \subseteq \mathbf{R}$ is a compact interval and $f: \Lambda \times X \rightarrow X$ is continuous and for each $\lambda \in \Lambda \quad f_{\lambda}: X \rightarrow X$ given by $f_{\lambda}(x):=f(\lambda, x)$ is a homeomorphism. For $\Delta \subseteq \Lambda$ consider the mapping

$$
f(\Delta): \Delta \times X \ni(\lambda, x) \rightarrow(\lambda, f(\lambda, x)) \in \Delta \times X .
$$

Note the following simple

Proposition 7.1. For every $\lambda \in \Delta$ the set $\{\lambda\} \times X$ is invariant with respect to $f(\Delta)$.

We will simply write $\lambda$ or $\Delta$ instead of $f_{\lambda}$ or $f(\Delta)$ in all cases where $f_{\lambda}$ or $f(\Delta)$ appears as a parameter.

Proposition 7.2. Assume $N \subseteq X$ is compact. Then the m.v. mappings

$$
\Lambda \ni \lambda \rightarrow \operatorname{Inv}^{-}(N, \lambda) \subseteq N, \quad \Lambda \ni \lambda \rightarrow \operatorname{Inv}^{+}(N, \lambda) \subseteq N
$$

are u.s.c.

Proof. Fix $\mu \in \Lambda$ and assume the mapping

$$
\lambda \rightarrow \operatorname{Inv}^{-}(N, \lambda)
$$

is not u.s.c. at $\mu$. Then there exist $U$ open in $N$ such that $\operatorname{Inv}^{-}(N, \mu) \subseteq U$ and a sequence $\left\{\lambda_{n}\right\} \subseteq \Lambda, \lambda_{n} \rightarrow \mu$, such that $\operatorname{Inv}^{-}\left(N, \lambda_{n}\right) \not \subset U$. Choose $z_{n} \in \operatorname{Inv}^{-}\left(N, \lambda_{n}\right) \backslash U$. Taking a subsequence, if necessary, we can assume that $z_{n} \rightarrow z$ for some $z \in N \backslash U$. Since $N$ is compact, we get from $\left(f_{\lambda_{n}}\right)^{-k}\left(z_{n}\right) \in N$ that $\left(f_{\mu}\right)^{-k}(z) \in N$ for all $k \in \mathbf{Z}^{+}$. Thus $z \in \operatorname{Inv}^{-}(N, \mu) \subseteq U$, a contradiction.

The proof of the remaining case is similar.

Corollary 7.3. If for some $\mu \in \Lambda \quad N$ is an isolating neighborhood with respect to $f_{\mu}$, then $N$ is an isolating neighborhood with respect to $f_{\lambda}$ for $\lambda$ sufficiently close to $\mu$.

Proof. We have $\operatorname{Inv}(N, \mu) \subseteq \operatorname{int} N$. Since

$$
\operatorname{Inv}(N, \mu)=\operatorname{Inv}^{-}(N, \mu) \cap \operatorname{Inv}^{+}(N, \mu),
$$

there exist open neighborhoods $U, V$ of $\operatorname{Inv}^{-}(N, \mu), \operatorname{Inv}^{+}(N, \mu)$, respectively, such that $U \cap V \subseteq$ int $N$. Using the above proposition one can find

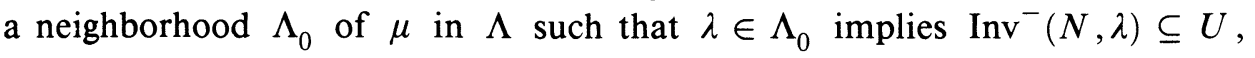


$\operatorname{Inv}^{+}(N, \lambda) \subseteq V$, i.e., $\operatorname{Inv}(N, \lambda) \subseteq U \cap V \subseteq \operatorname{int} N$. This shows that $N$ is an isolating neighborhood with respect to $f_{\lambda}$ for $\lambda \in \Lambda_{0}$.

Lemma 7.4. Assume that $N$ is an isolating neighborhood with respect to $f_{\mu}$ for some $\mu \in \Lambda$. If $P, Q \in \operatorname{IP}(N, \mu)$ are such that $P \subseteq$ int $_{N} Q$ and $P$ is related to $Q$, then there exists $\Lambda_{0}$, a neighborhood of $\mu$ in $\Lambda$, such that for every $\lambda \in \Lambda_{0}$ there exists $P(\lambda) \in \operatorname{IP}(N, \lambda)$ satisfying $P \subseteq P(\lambda) \subseteq Q$.

Proof. Put $P(\lambda):=F d_{N, \lambda}(P)$; we will show that for $\lambda$ sufficiently close to $\mu$ the index pair $P(\lambda)$ satisfies the assertion of the lemma.

Put $Z:=N \backslash$ int $_{N} Q_{1}$. By Proposition 7.2 and Corollary 7.3 one can find a compact neighborhood $\Delta$ of $\mu$ in $\Lambda$ such that $N$ is an isolating neighborhood with respect to $f_{\lambda}$ and

$$
\operatorname{Inv}^{-}(N, \lambda) \subseteq \operatorname{int}_{N} P_{1}, \quad \operatorname{Inv}^{+}(N, \lambda) \subseteq N \backslash Q_{2} \quad \text { for all } \lambda \in \Delta .
$$

In particular,

$$
\operatorname{Inv}^{-}(N, \lambda) \cap Z=\varnothing, \quad \operatorname{Inv}^{+}(N, \lambda) \cap P_{2}=\varnothing \quad \text { for } \lambda \in \Delta,
$$

because $P \subseteq$ int $_{N} Q$. Put $g:=f(\Delta), M:=N \times \Delta$. One can easily verify that $M$ is an isolating neighborhood with respect to $g$ and

$$
\begin{aligned}
& \operatorname{Inv}^{-}(M, g) \cap Z \times \Delta=\bigcup\left\{\operatorname{Inv}^{-}(N, \lambda) \cap Z \times\{\lambda\} \mid \lambda \in \Lambda\right\}=\varnothing, \\
& \operatorname{Inv}^{+}(M, g) \cap P_{2} \times \Delta=\bigcup\left\{\operatorname{Inv}^{+}(N, \lambda) \cap P_{2} \times\{\lambda\} \mid \lambda \in \Lambda\right\}=\varnothing .
\end{aligned}
$$

Let $x \in Z$. Then $B k_{N, \mu}(x) \cap P_{1}=\varnothing$ and $(x, \mu) \in Z \times \Delta$, i.e., $B k_{M, g}(x, \mu) \cap$ $P_{1} \times \Delta=\varnothing$. Thus it follows from the upper semicontinuity of $B k_{M, g}$ (see Lemma 5.5) that there exists an open neighborhood $V_{x}$ of $x$ in $N$ and $\Delta_{x}$ of $\mu$ in $\Delta$ such that

$$
B k_{M, g}(y, \lambda) \cap P_{1} \times \Delta=\varnothing \quad \text { for } y \in V_{x}, \lambda \in \Delta_{x},
$$

i.e.,

$$
B k_{N, \lambda}(y) \cap P_{1}=\varnothing \quad \text { for } y \in V_{x}, \lambda \in \Delta_{x} \text {. }
$$

A compactness argument proves that there exists a finite subset $Z_{0} \subseteq Z$ such that $Z \subseteq \bigcup\left\{V_{x} \mid x \in z_{0}\right\}$. Put $\Delta_{0}:=\bigcap\left\{\Delta_{x} \mid x \in Z_{0}\right\}$. Then $\Delta_{0}$ is a neighborhood of $\mu$ and $B k_{N, \lambda}(y) \cap P_{1}=\varnothing$ for $y \in Z, \lambda \in \Delta_{0}$. This shows that $P_{1}(\lambda)=F d_{N, \lambda}\left(P_{1}\right) \subseteq N \backslash Z=$ int $_{N} Q_{1} \subseteq Q_{1}$ for $\lambda \in \Delta_{0}$.

Now take $x \in P_{2}$. Then $F d_{N, \mu}(x) \subseteq P_{2} \subseteq$ int $Q_{2}$. Similar reasoning using a compactness argument and the mapping $g$ shows that there exists a neighborhood $\Delta_{1}$ of $\mu$ such that $F d_{N, \lambda}(y) \subseteq$ int $_{N} Q_{2}$ for $y \in P_{2}$ and $\lambda \in \Delta_{1}$. Thus $P_{2}(\lambda)=F d_{N, \lambda}\left(P_{2}\right) \subseteq Q_{2}$ for $\lambda \in \Delta_{1}$.

It follows from Lemmas 5.6, 5.7 that $P_{1}(\lambda)$ and $P_{2}(\lambda)$ are compact. Obviously they are positively invariant with respect to $f_{\lambda}$ and $P_{i} \subseteq P_{i}(\lambda)$ for $i=1,2$. Thus we get from (7.1) that

$$
\operatorname{Inv}^{-}(N, \lambda) \subseteq \operatorname{int}_{N} P_{1} \subseteq \operatorname{int}_{N} P_{1}(\lambda), \quad \operatorname{Inv}^{+}(N, \lambda) \subseteq N \backslash Q_{2} \subseteq N \backslash P_{2}(\lambda),
$$


for $\lambda \in \Delta$. A compactness argument with respect to $\operatorname{cl}\left(Q_{1} \backslash P_{2}\right)$ shows that for $\lambda$ in some neighborhood of $\mu$ we have

$$
P_{1}(\lambda) \backslash P_{2}(\lambda) \subseteq Q_{1} \backslash P_{2} \subseteq \text { int } N \cap f_{\lambda}^{-1}(\text { int } N)
$$

Hence $P(\lambda) \in \operatorname{IP}(N, \lambda)$ for $\lambda$ sufficiently close to $\mu$.

The following lemma strengthens the previous result.

Lemma 7.5. Assume $N \subseteq X, \mu \in \Lambda, P, Q \in \operatorname{IP}(N, \mu)$ are as in the previous lemma. Then there exists $\Lambda_{0}$ a neighborhood of $\mu$ in $\Lambda$, such that for every $\kappa \in \Lambda_{0}$ there exists $R(\kappa) \in \operatorname{IP}(N, \kappa)$ satisfying $P \subseteq R(\kappa) \subseteq Q$ and such that the inclusions

$$
i: P \rightarrow R(\kappa), \quad j: R(\kappa) \rightarrow Q
$$

induce morphisms

$$
\begin{aligned}
& i^{*}:\left(H^{*}(R(\kappa)), I_{R(\kappa)}\right) \rightarrow\left(H^{*}(P), I_{P}\right), \\
& j^{*}:\left(H^{*}(Q), I_{Q}\right) \rightarrow\left(H^{*}(R(\kappa)), I_{R(\kappa)}\right) .
\end{aligned}
$$

Proof. Applying Lemma 5.15, find $R, S \in \operatorname{IP}(N)$ such that $P \subseteq$ int $_{N} R \subseteq$ $R \subseteq$ int $_{N} S \subseteq S \subseteq$ int $_{N} Q$. It follows from Lemma 7.4 that we can find a neighborhood $\Lambda_{0}$ of $\mu$ such that for every $\lambda \in \Lambda_{0}$ there exist index pairs $P(\lambda), R(\lambda), S(\lambda)$ satisfying

$$
P \subseteq P(\lambda) \subseteq R \subseteq R(\lambda) \subseteq S \subseteq S(\lambda) \subseteq Q
$$

Fix $\kappa \in \Lambda_{0}$. To show that $R(\kappa)$ satisfies the assertion of the lemma consider the following, generally noncommutative diagram

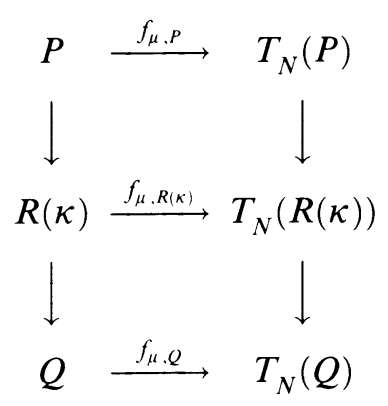

in which vertical arrows denote inclusions. The diagram is homotopy commutative, because for any $\lambda \in \Lambda_{0}$

$$
\begin{gathered}
f_{\lambda}(P) \subseteq f_{\lambda}(P(\lambda)) \subseteq T_{N}(P(\lambda)) \subseteq T_{N}(R) \subseteq T_{N}(R(\kappa)) \\
f_{\lambda}(R(\kappa)) \subseteq f_{\lambda}(S) \subseteq f_{\lambda}(S(\lambda)) \subseteq T_{N}(S(\lambda)) \subseteq T_{N}(Q)
\end{gathered}
$$


and $\mu, \kappa$ are joined in $\Lambda_{0}$ by an arc. Thus we get the following commutative diagram in the Alexander-Spanier cohomology.

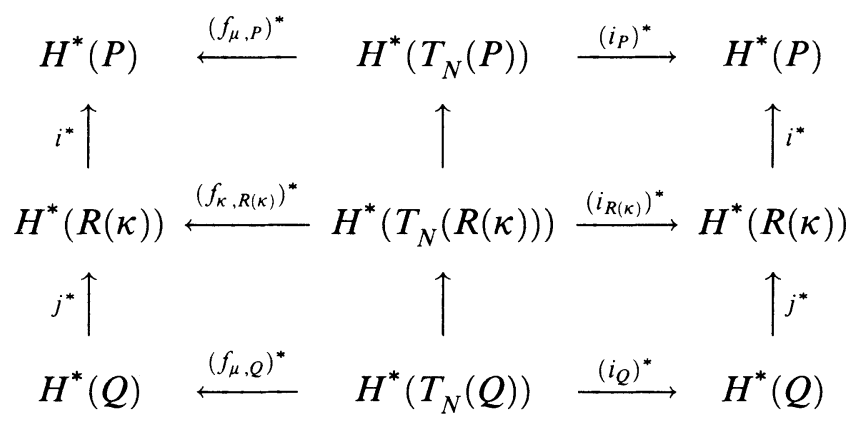

which, in view of the definition of an index map (see Definition 2.5), finishes the proof.

Proof of Theorem 2.11. Obviously it is sufficient to show that for any $\mu \in \Lambda$ there exists $\Lambda_{0}$, a neighborhood of $\mu$, such that for all $\nu \in \Lambda_{0}$

$$
C(\operatorname{Inv}(N, \mu))=C(\operatorname{Inv}(N, \nu)) .
$$

Thus fix $\mu \in \Lambda$ and using Lemmas 5.12 and 5.15 find index pairs $P, Q, R \in$ $\operatorname{IP}(N, \mu)$ such that $P \subseteq$ int $_{N} Q, Q \subseteq$ int $_{N} R, P$ is related to $Q, Q$ is related to $R$. Applying Lemma 7.5 twice we can find $\Lambda_{0}$, a neighborhood of $\mu$ in $\Lambda$, such that for every $\lambda \in \Lambda_{0}$ there exist index pairs $P(\lambda), Q(\lambda) \in \operatorname{IP}(N, \lambda)$ satisfying $P \subseteq P(\lambda) \subseteq Q \subseteq Q(\lambda)$ and such that we have the following commutative diagram of maps induced by inclusions:

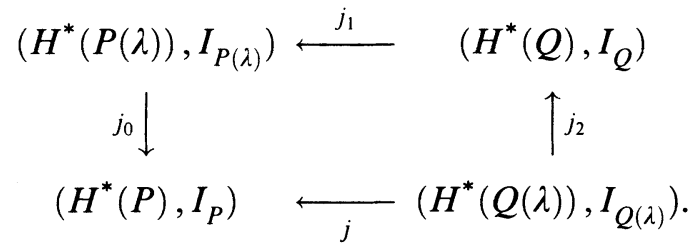

Applying the Leray functor to the above diagram we get from Theorem 6.4 that $L\left(j_{0}\right) \circ L\left(j_{1}\right)=L\left(j_{0} \circ j_{1}\right), L\left(j_{1}\right) \circ L\left(j_{2}\right)=L\left(j_{1} \circ j_{2}\right)$ are isomorphisms; thus $L(j)$ is also an isomorphism. Hence

$$
C(\operatorname{Inv}(N, \mu))=L\left(H^{*}(P), I_{P}\right)=L\left(H^{*}\left(Q(\lambda), I_{Q(\lambda)}\right)=C(\operatorname{Inv}(N, \lambda)),\right.
$$

which finishes the proof.

Recall that for $P \in \operatorname{IP}(N) S(P):=\left(P_{1} \cup f\left(P_{2}\right), P_{2} \cup f\left(P_{2}\right)\right)$. Property (2.8) and the commutative diagram

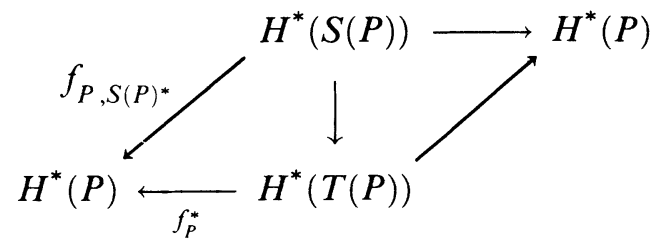

in which the unmarked arrows indicate maps induced by inclusions, imply the following proposition. 

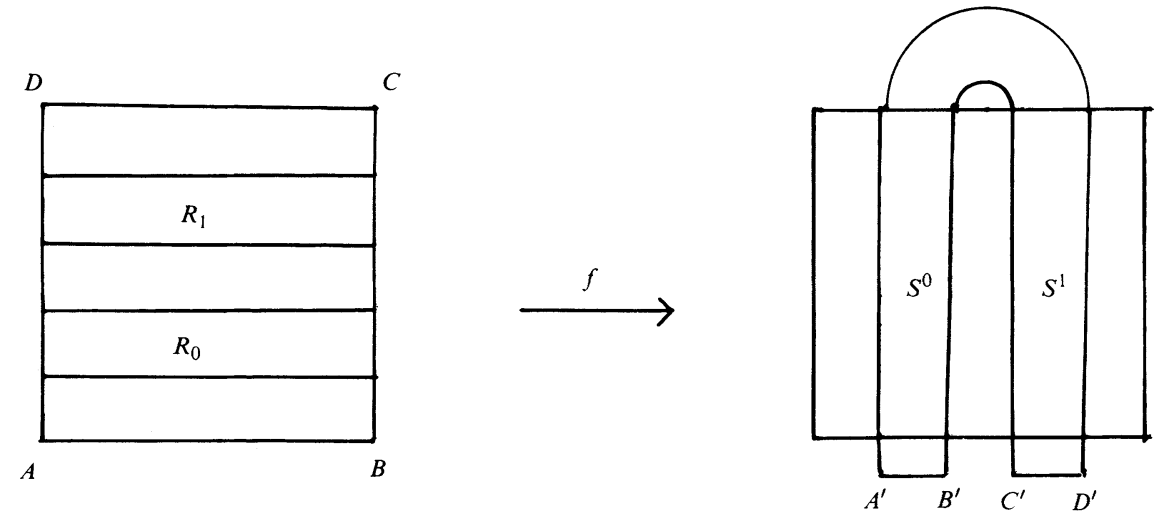

FIGURE 1

Proposition 7.6. For every $P \in \operatorname{IP}(N)$

$$
I_{P}=f_{P, S(P)}^{*} \circ i_{P, S(P)}^{*-1} \text {. }
$$

Proof of Theorem 2.12. Choose $U_{1}, U_{2}$ to be disjoint, open neighborhoods of $K_{1}$ and $K_{2}$, respectively. For $i=1,2$ let $N_{i}$ be a compact neighborhood of $K_{i}$ such that

$$
N_{i} \subseteq U_{i} \cap f^{-1}\left(U_{i}\right) .
$$

Select $P^{\prime}, P^{\prime \prime}$ index pairs of $K_{1}$ and $K_{2}$ in $N_{1}$ and $N_{2}$, respectively. One can easily verify that $P:=P^{\prime} \cup P^{\prime \prime}$ is an index pair of $K$ in $N:=N_{1} \cup N_{2}$. It follows from (7.2) that $S\left(P^{\prime}\right) \cap S\left(P^{\prime \prime}\right)=\varnothing$; thus $H^{*}(S(P))=H^{*}\left(S\left(P^{\prime}\right)\right) \times H^{*}\left(S\left(P^{\prime \prime}\right)\right)$. Obviously also $H^{*}(P)=H^{*}\left(P^{\prime}\right) \times H^{*}\left(P^{\prime \prime}\right)$ and, by Proposition 7.6,

$$
\begin{aligned}
I_{P} & =f_{P, S(P)}^{*} \circ i_{P, S(P)}^{*-1} \\
& =\left(f_{P^{\prime}, S\left(P^{\prime}\right)}^{*} \times f_{P^{\prime \prime}, S\left(P^{\prime \prime}\right)}^{*}\right) \circ\left(i_{P^{\prime}, S\left(P^{\prime}\right)}^{*-1} \times i_{P^{\prime \prime}, S\left(P^{\prime \prime}\right)}^{*-1}\right) \\
& =\left(f_{P^{\prime}, S\left(P^{\prime}\right)}^{*} \circ i_{P^{\prime}, S\left(P^{\prime}\right)}^{*-1}\right) \times\left(f_{P^{\prime \prime}, S\left(P^{\prime \prime}\right)}^{*} \circ i_{P^{\prime \prime}, S\left(P^{\prime \prime}\right)}^{*-1}\right) \\
& =I_{P^{\prime}} \times I_{P^{\prime \prime}} .
\end{aligned}
$$

Thus $\left(H^{*}(P), I_{P}\right)=\left(H^{*}\left(P^{\prime}\right), I_{P^{\prime}}\right) \times\left(H^{*}\left(P^{\prime \prime}\right), I_{P^{\prime \prime}}\right)$. Now we get from Proposition 4.5

$C(K)=L\left(H^{*}(P), I_{P}\right)=L\left(H^{*}\left(P^{\prime}\right), I_{P^{\prime}}\right) \times L\left(H^{*}\left(P^{\prime \prime}\right), I_{P^{\prime \prime}}\right)=C\left(K_{1}\right) \times C\left(K_{2}\right)$.

\section{EXAMPLES AND FINAL REMARKS}

In this section we take the coefficient ring $\Xi$ to be $\mathbf{Q}$, the field of rational numbers. We begin with computing the index of some horseshoes.

Example 8.1 (index of horseshoes). Assume $X=S^{2}=\mathbf{R} \cup\{\infty\}$ and $f: X \rightarrow X$ is a continuous map such that $f$ maps two rectangles $R_{0}$ and $R_{1}$ linearly into 

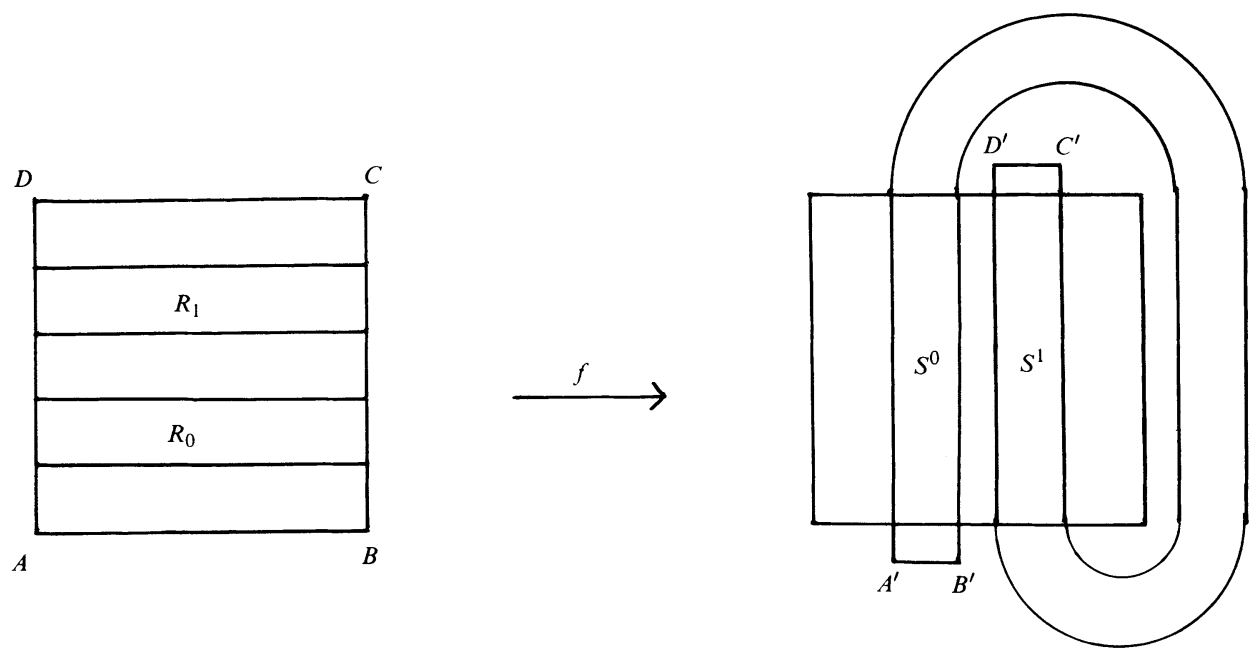

FIGURE 2

rectangles $S^{0}$ and $S^{1}$ as indicated in Figure 1. Then $N:=[0,5] \times[0,5]$ is an isolating neighborhood. Take

$$
P_{1}:=[1,4] \times[0,5], \quad P_{2}:=[1,4] \times([0,1] \cup[2,3] \cup[4,5]) .
$$

Then $P:=\left(P_{1}, P_{2}\right) \in \operatorname{IP}(N, f), H^{*}(P)$ is a free module with two generators $\alpha, \beta$, and $I_{P}$ (up to a permutation of $\alpha, \beta$ ) has the matrix

$$
\left[\begin{array}{ll}
1 & -1 \\
1 & -1
\end{array}\right]
$$

It follows that $I_{P}^{2}=0, H^{*}(P) / \operatorname{gker}\left(I_{P}\right)=0$, and consequently the Conley index of the classical Smale's horseshoe is zero.

This is not the case for the isolated invariant set $K$ of the horseshoe on Figure 2. $P$ as above is again an index pair but $I_{P}$ has the matrix

$$
\left[\begin{array}{ll}
1 & 1 \\
1 & 1
\end{array}\right] \text {. }
$$

Since $I_{P}^{2}=2 I_{P}$, it follows that $\operatorname{gker}\left(I_{P}\right)=\operatorname{ker}\left(I_{P}\right)$ and we find that

$$
C_{k}(K)= \begin{cases}0, & k \neq 1, \\ (\mathbf{Q}, 2 \text { id }), & k=1 .\end{cases}
$$

However, one should note that similar computations for integer coefficients give a zero index, because $2 \mathrm{id}: \mathbf{Z} \rightarrow \mathbf{Z}$ is not an isomorphism, only a monomorphism and its generalized image is zero. This shows that sometimes rational coefficients may provide more information (unlike the case of homology theory).

As in the two other cases, one can find that the index of the isolated invariant set $K$ of the horseshoe on Figure 3 is

$$
C_{k}(K)= \begin{cases}0, & k \neq 1, \\ (\mathbf{Q}, \text { id }), & k=1 .\end{cases}
$$



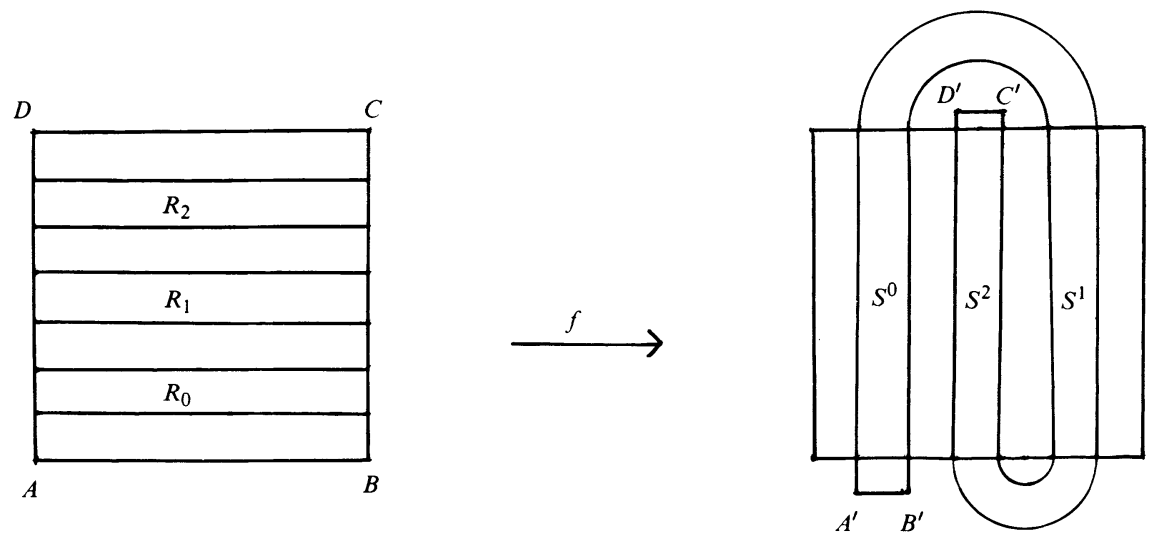

FigURE 3

Hence for the three horseshoes we obtain three different indices. It follows that none of them can be deformed into another one without giving rise to bounded trajectories crossing the boundary of $N$. Otherwise we would contradict the homotopy invariance of the index.

Example 8.2 (a hyperbolic circle). Consider the map

$$
f: S^{1} \times \mathbf{R} \ni(z, x) \rightarrow(a z,-2 x) \in S_{1} \times \mathbf{R},
$$

where $S^{1}$ is the unit circle of the complex plane and $a \in S^{1}$ is fixed. The map has an invariant isolated circle, namely $S^{1} \times\{0\}$. Take

$$
\begin{aligned}
& N:=P_{1}:=\left\{(z, x) \in S^{1} \times \mathbf{R}|| x \mid \leq 2\right\}, \\
& P_{2}:=\{(z, x) \in N|| x \mid \geq 1\} .
\end{aligned}
$$

It is straightforward to verify that $P:=\left(P_{1}, P_{2}\right) \in \operatorname{IP}(N, f)$ and

$$
H^{k}(P)= \begin{cases}\mathbf{Q} & \text { for } k=1 \text { or } k=2, \\ 0 & \text { otherwise. }\end{cases}
$$

Less obvious, though not very difficult, is to see that $I_{P}(\alpha)=-\alpha, I_{P}(\beta)=$ - $\beta$ for both generators $\alpha \in H^{1}(P), \beta \in H^{2}(P)$. It follows that $I_{P}$ is an isomorphism and $C\left(S^{1} \times\{0\}\right)=\left(H^{*}(P), I_{P}\right)$.

Example 8.3. Let $\Lambda \subseteq \mathbf{R}$ be a compact interval and let $f_{\lambda}: \mathbf{R}^{n} \rightarrow \mathbf{R}^{n}$ be a family of diffeomorphisms with continuous dependence on $(\lambda, x) \in \Lambda \times X$. Assume for $\lambda \in \Lambda D_{\lambda}=\left\{u_{\lambda}, v_{\lambda}\right\}$ is an invariant set with respect to $f_{\lambda}$ such that for some $\mu \in \Lambda$

(8.1) $u_{\mu}=v_{\mu}, u_{\lambda} \neq v_{\lambda}$ for $\lambda \neq \mu$,

(8.2) $u_{\lambda}, v_{\lambda}$ are two hyperbolic fixed points of $f_{\lambda}$ with no negative eigenvalues for $\lambda<\mu$,

(8.3) $\left\{u_{\lambda}, v_{\lambda}\right\}$ is a hyperbolic 2-periodic orbit of $f_{\lambda}$ for $\lambda>\mu$. 
Then there exists a neighborhood $V$ of $D_{\mu}$ such that for some $\lambda$ sufficiently close to $\mu$ there are bounded trajectories of $f_{\lambda}$ contained in $V$ but disjoint from $D_{\lambda}$. In other words, contrary to the case of a flow (see [Co, Example I.4.5]), a hyperbolic periodic solution of a discrete dynamical system cannot be continued to two disjoint hyperbolic fixed points without giving rise to some other bounded trajectories.

To see this, assume the contrary. Then the index of two hyperbolic fixed points would equal the index of the hyperbolic periodic orbit. This is, however, impossible, because the index map maps one generator into the other (up to the sign) in case of the periodic orbit and into the same in case of two periodic points.

Example 8.4. C. Conley proved in $[\mathrm{Co}, \S 1.5]$ that the system of differential equations

$$
\left\{\begin{array}{l}
x_{i}^{\prime}=x_{i+1}, \quad i=1,2, \ldots, n-1, \\
x_{n}^{\prime}=\left(x_{1}\right)^{2}-1
\end{array}\right.
$$

admits a nonconstant, bounded solution. A natural question is whether the same applies to the discrete-time dynamical system obtained from (8.4) by the Euler approximation method, i.e., to the system defined by the map

$$
f_{s}: \mathbf{R}^{n} \ni\left(x_{1}, x_{2}, \ldots, x_{n}\right) \rightarrow\left(x_{1}, x_{2}, \ldots, x_{n}\right)+s\left(x_{2}, \ldots, x_{n},\left(x_{1}\right)^{2}-1\right) \in \mathbf{R}^{n},
$$

where $s$ is a constant denoting the step of the Euler method. After the linear change of coordinates $z_{j}:=s^{n-1+j} x_{j}$ the map $f_{s}$ becomes

$$
f_{s}: \mathbf{R}^{n} \ni\left(z_{1}, z_{2}, \ldots, z_{n}\right) \rightarrow\left(z_{1}, z_{2}, \ldots, z_{n}\right)+\left(z_{2}, \ldots, z_{n},\left(z_{1}\right)^{2}-s^{2 n}\right) \in \mathbf{R}^{n} .
$$

As in Conley's proof concerning (8.4), one can show that the origin is the only nontrivial isolated invariant set with respect to the map $f_{0}$ and $C\left(\{0\}, f_{0}\right)=0$. Hence it follows that $B:=\left\{x \in \mathbf{R}^{n}:\|x\| \leq 1\right\}$ is an isolating neighborhood with respect to $f_{s}$ for small $s$. If the two fixed points of $f_{s}$ were the only trajectories of $f_{s}$ in $B$, then, by Theorem 2.12, they would both have zero index. However, an easy computation shows that at least one of the fixed points is hyperbolic; thus, by Theorem 3.1, at least one of them has nonzero index.

This shows that the Euler approximation of the system (8.4) also admits a nonconstant, bounded trajectory at least for small values of the step $s$.

In this paper we proposed an alternative approach to the Conley index for discrete dynamical systems. Obviously, several questions are left open. The first question is, what is the formal relation between our index and the shape index? Cohomology carries less information than shape; hence in this respect our index is less general than the shape index. On the other hand, as we have seen, much information is contained in the distinguished isomorphism and this information is lost in case of the shape index. Nevertheless, some kind of formal relation between both indices seems to exist. 
Another question is whether all objects of $\mathscr{E} I$ (all isomorphisms) can be realized. It appears not, at least in zero dimension.

In the detailed study of isolated invariant sets as in Example 8.2 it would be convenient to have a product formula for the index as in the continuous case (see [Co, I.4.2]). Such a product formula for discrete dynamical systems can be formulated and proved at least in the case of field coefficients and isolated invariant sets with indices of finite type (i.e., with finite numbers of generators). This suffices for many applications. We shall discuss this in detail in a separate paper, because it is not yet clear to the author whether the finite-type assumption is essential. To answer this question one requires further studies of the properties of the Leray functor.

An important problem is what generalizations of the presented index are possible. As we already mentioned, contrary to the shape index, the discrete semidynamical system (continuous map) on a locally compact space can be treated just as in this paper, only with obvious changes in definitions. This is important, because the discretization of a differential equation is usually not injective. In the forthcoming paper [MR] continuous mappings in nonlocally compact spaces will also be considered.

Attractor-repeller pairs and the Morse-Smale equation for the discrete case can be studied similarly to the continuous case. Details will be presented in [Mr3]. One would like to know, however, whether such notions of the classical Conley index theory as connected simple system index (see [Ku]), connection index (see [Co, III.7.2]), index braids, and connection matrices (see [Fr1, Fr2]) can be included in the presented case. Hence there is a broad field for future investigations.

Acknowledgments. I express my gratitude to Stanisław Sȩdziwy and Roman Srzednicki for inspiration, discussions and comments enhancing the presentation of this paper. It is also my pleasure to thank Bernold Fiedler and an anonymous referee for suggesting some interesting examples.

\section{REFERENCES}

[Co] C. C. Conley Isolated invariant sets and the Morse index, CBMS Regional Conf. Ser. in Math., no. 38, Amer. Math. Soc., Providence, R.I., 1978.

[Fr1] R. Franzosa, Index filtrations and the homology index braid for partially ordered Morse decompositions, Trans. Amer. Math. Soc. 298 (1986), 193-213.

[Fr2] _ The connection matrix theory for Morse decompositions, Trans. Amer. Math. Soc. 311 (1989), 561-592.

[Ku] H. L. Kurland, The Morse index of an isolated invariant set is a connected simple system, $\mathbf{J}$. Differential Equations 42 (1981), 234-259.

[Le] J. Leray, Théories des points fixes: indice total à nombre de Lefschetz, Bull. Soc. Math. France 87 (1959), 221-233.

[Mr1] M. Mrozek, Index pairs and the fixed point index for semidynamical systems with discrete time, Fund. Math. (in press).

[Mr2] __ The cohomological index of Conley type for multi-valued admissible flow's, J. Differential Equations (in press). 
[Mr3] _ , The Morse equation in Conley's index theory for homeomorphisms, Topology Appl. (to appear).

[MR] M. Mrozek and K. P. Rybakowski, A cohomological conley index for discrete time dynamical systems (to appear).

[Ni] Z. Nitecki, Differentiable dynamics, an introduction to the orbit structure of diffeomorphisms, MIT Press, Cambridge, Mass., and London, 1971.

[RS] J. W. Robbin and D. Salamon, Dynamical systems, shape theory and the Conley index, Ergodic Theory Dynamical Systems 8* (1988), 375-393.

[Ry] K. Rybakowski, On the homotopy index of infinite dimensional semiflows, Trans. Amer. Math. Soc. 269 (1982), 351-382.

[Sp] E. H. Spanier, Algebraic topology, McGraw-Hill, New York, 1966.

Department of Computer Science, Jagiellonian University, 31-501 Kraków, Poland 